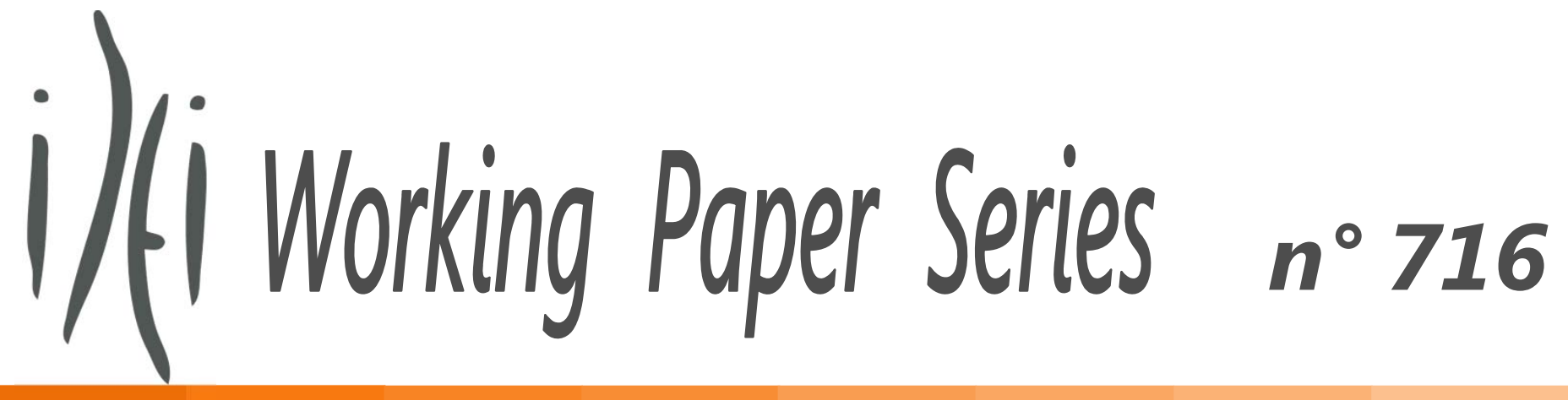

May, 2012

\title{
" Voting Power in the EU Council of Ministers and Fair Decision Making in Distributive Politics"
}

\author{
Michel Le Breton
}

Maria Montero

and

Vera Zaporozhets 


\title{
Voting Power in the EU Council of Ministers and Fair Decision Making in Distributive Politics*
}

\author{
Michel Le Breton
}

\author{
Maria Montero
}

May 2012

\author{
Vera Zaporozhets
}

\begin{abstract}
We analyze and evaluate the different decision rules describing the Council of Ministers of the EU starting from 1958 up to now. Most of the existing studies use the Banzhaf index (for binary voting) or the Shapley-Shubik index (for distributive politics). We argue in favor of the nucleolus as a power measure in distributive situations and an alternative to the Shapley-Shubik index. We then calculate the nucleolus and compare the results of our calculations with the conventional measures. In the second part, we analyze the power of the European citizens as measured by the nucleolus under the egalitarian criterion proposed by Felsenthal and Machover (1998), and characterize the first best situation. Based on these results we propose a methodology for the design of the optimal (fair) decision rules. We perform the optimization exercise for the earlier stages of the EU within a restricted domain of voting rules, and conclude that Germany should receive more than the other three large countries under the optimal voting rule.
\end{abstract}

Keywords: voting power, distributive politics, nucleolus, constitutional design.

J.E.L. classification numbers: C71, C72, C78, D63, D72.

*We would like to thank Ruslan Sadykov for help with the EU27 computations. We would also like to thank Moshé Machover, Stefan Napel, Josep Freixas, Serguei Kaniovski, Sascha Kurz, two anonymous referees and participants in the 10th international meeting of the society for Social Choice and Welfare and in the 2011 ESEM-EEA meetings for helpful comments. 


\section{Introduction}

Democratic decision-making is based on voting. Political scientists and economists have long noted that it is far from obvious how to measure the voting power of different individuals or groups, e.g. parliamentary coalitions, in decision-making bodies. They noticed that voting power is not always proportional to the number of votes controlled by the individual or group. For example, Luxembourg was powerless in the Council of Ministers of the EU between 1958 and 1973. It held one vote, whereas a qualified majority of votes was defined to be 12 out of 17. Since all other member states held an even number of votes, Luxembourg formally was never able to make a difference. The recent enlargement of the European Union caused a lively debate on the adequate tools for measuring power and had strong implications for the balance of power among member states.

There is a vast theoretical and empirical literature on power measures. ${ }^{1}$ Most of this literature assumes that the decision that must be made by the committee is a binary yes/no decision on an external proposal; compromise solutions or other amendments to the proposal are not possible. The power of a voter in this binary setting is usually measured by the probability of the voter being pivotal. For example the Penrose (1946) measure, of which the Banzhaf index is a normalization, can be interpreted as the probability of being pivotal when each member of the committee is equally likely to vote yes and no, and all committee members vote independently.

There is less consensus as to the best way to measure power when the decision is not binary. Napel and Widgrén (2004) note that

"Scientists who study power ... seem divided into two disjoint methodological camps. The first one uses non cooperative game theory to analyze the impact of explicit decision making procedures and given preferences over a well-defined, usually Euclidean policy space. The second one stands in the tradition of cooperative game theory with more abstractly defined voting bodies: the considered agents have no particular preferences and form winning coalitions which implement unspecified policies. Individual chances of being part of and influencing a winning coalition are then measured by a power index... Several authors have concluded that it is time to develop a unified framework for measuring decision power. On the one hand, such framework should allow for predictions and ex post analysis of decisions based on knowledge of procedures and preferences. On the other hand, it must be open to ex ante and even completely a priori analysis of power when detailed information may either not be available or should be ignored for normative reasons".

This approach is followed by Steunenberg, Schmidtchen and Koboldt (1999) and Napel and Widgrén (2004). They assume a multidimensional policy space ${ }^{2}$ and a mapping from

\footnotetext{
${ }^{1}$ Recent papers include Algaba et al. (2007), Barr and Passarelli (2009), Bilbao et al. (2002), Felsenthal and Machover (2001, 2004), Kaniovski and Leech (2009), Laruelle and Widgrén (1998) and Leech (2002).

${ }^{2}$ Other papers departing from the binary setting are Maaser and Napel (2007) and Kurz et al. (2011) who consider a unidimensional policy space, and Laruelle and Valenciano (2008, chapter 4), who consider a multidimensional policy space.
} 
the ideal points of the voters and the status quo to an outcome (usually the equilibrium of a noncooperative game $)^{3}$; the ideal points and status quo are randomly determined according to some probability distribution. The strategic power index of Steunenberg, Schmidtchen and Koboldt (1999) is based on the expected distance between a player's ideal point and the equilibrium outcome, with a larger distance indicating a less powerful player; a dummy player who has no voting rights is used as the benchmark for comparison. Napel and Widgrén (2004) compare the actual outcome with a shadow outcome that would arise if all other voters keep their strategy but the voter in question deviates from equilibrium; power is measured by the sensitivity of the outcome to the actions of that voter.

Our paper focuses on surplus distribution, so we consider a specific, but extremely important, multidimensional policy space, namely the simplex of some Euclidean space. This setting arises naturally when the issue under scrutiny is the allocation of a fixed budget across the members of an organization. More generally, under the assumptions of transferable utility (i.e., quasi-linearity with respect to some common numeraire) and efficiency in public decision making, the simplex structure appears as the efficient frontier of any bounded and convex subset of policies like those considered in spatial models of politics. The key assumption we make on preferences is that members care only about their share. Their ideal points are the vertices of the simplex and there is no room for a difference between ex ante and ex post power measurement from the perspective of preferences.

Given the policy space we consider, the probability of being pivotal is a very imprecise measure of voting power. ${ }^{4}$ For example, if the final outcome is the distribution of the surplus within a minimal winning coalition, all members of the coalition are presumably pivotal, but some members may receive very little whereas others may receive a lot. These different shares are the result of differences in power, and thus we chose to measure power by the expected payoff of a voter ${ }^{5,6}$.

The question arises of what measure of expected payoff to use. We certainly agree with Napel and Widgrén's postulate that game forms have to be taken into account by political analysis but we do not want the power analysis to be extremely sensitive to the details of the game form used to describe the non-cooperative decision process, i.e. we would like a robust power measure. We have chosen the nucleolus (Schmeidler, 1969) because, as we will discuss

\footnotetext{
${ }^{3}$ Several authors including Napel and Widgrén $(2006,2011)$ and Tsebelis (1994) have analyzed noncooperative game forms describing the interaction between different EU decision making bodies.

${ }^{4}$ As we will see in section 2, all voters may have the same probability of being pivotal even if there are large asymmetries between them.

${ }^{5}$ Early studies defined success (or satisfaction) as power + luck in the binary setting. Success means getting what one voted for. This may be due to power (being pivotal) or luck (e.g., having the same preferences as another player who is pivotal). Because voters' preferences are completely opposed in our setting, luck plays no role and any positive payoff is a reflection of power.

${ }^{6}$ The notion of power as expected payoff is far from new. Felsenthal and Machover (1998) discuss this notion and call it P-power, as opposed to the probability of being pivotal in binary voting, which they call I-power. The Shapley value, of which the Shapley-Shubik index is a special case, can be interpreted as the expected payoff of playing a cooperative game (see Shapley (1953) and Roth (1977)). Expected payoffs play a central role in the strategic power index of Steunenberg, Schmidtchen and Koboldt (1999), though power in their setting needs to be disentangled from luck (see Napel and Widgrén (2004); Schmidtchen and Steunenberg (2011)).
} 
in section 2, it appears as the vector of equilibrium payoffs for the voters in two unrelated games that have been used to model the public decision making process. The first is the legislative bargaining game with random proposers of Baron and Ferejohn (1989), in which voters directly make proposals and vote over how to divide a budget between themselves. If proposal probabilities coincide with the nucleolus, then the nucleolus is the unique vector of expected payoffs (Montero, 2006); the nucleolus may arise for other proposal probabilities as well depending on the voting game. The second is the sequential lobbying model of Groseclose and Snyder (1996), in which the legislature votes over a binary issue and two opposing lobbies buy the votes of (some of) the members of the legislature in order to get them to vote for their preferred alternative. If at equilibrium lobbying takes place, then the nucleolus is a vector of equilibrium payoffs and, often, the unique vector of equilibrium payoffs (see Young (1978 a,b), Le Breton and Zaporozhets (2010) and Le Breton, Sudhölter and Zaporozhets (2010)). In both models, the ex ante approach is well defined. In the bargaining model, it arises because the proposer is randomly selected. In the lobbying model, as suggested in Diermeier and Myerson (1999), randomness results from the fact that the willingness to pay of each lobby is the realization of a random variable and that lobbying takes place iff the ratio of the two realizations is larger than some threshold called the hurdle factor.

One of the important questions in the literature is whether the allocation of votes among EU states is fair, and in particular whether the large countries are under-represented relatively to the small ones. We address this question using the nucleolus as a power measure. In the first part of our paper, we analyze the distribution of power in the Council of Ministers according to the nucleolus starting from 1958 up to now. In the second part, we move to a normative analysis, i.e. to the determination of the weights that should be assigned to the members of the EU Council of Ministers in order to achieve a certain social objective. Hereafter, we will refer to these weights as being the optimal weights.

The question of finding the optimal weights has been addressed before in the literature, mostly in the binary setting in which an alternative is pitted against the status quo, rather than in a distributive setting in which the set of alternatives is a simplex ${ }^{7}$. Within the binary setting, there have been egalitarian approaches that seek to equalize the power of all citizens (as measured by the Banzhaf index), and utilitarian approaches that seek to maximize the total utility of all citizens. ${ }^{8}$ In this paper, we follow the egalitarian approach with the nucleolus being the measure of power of the countries in the EU Council of Ministers ${ }^{9}$ : in our setting the role of the Council of Ministers is to distribute some surplus across the countries. The country amount is then divided equally among their citizens (we do not introduce any bias). If this surplus is interpreted as the gains from the EU, we would like this surplus to be shared equally among European citizens ${ }^{10}$. It follows from our result that

\footnotetext{
${ }^{7}$ An exception is Laruelle and Valenciano (2008, chapter 4), who study the optimal voting rule in a generalized Nash bargaining setting.

${ }^{8}$ The egalitarian approach is implicit in Penrose's (1946) square root rule. The utilitarian approach has been explored by Beisbart, Bovens and Hartmann (2005), Barberà and Jackson (2006) and Beisbart and Hartmann (2010).

${ }^{9}$ As it will be clear, we could of course reproduce the analysis for any other power measure.

${ }^{10}$ The principle of "one person, one vote" is generally taken to be the cornerstone of democracy. In this distributive setting, the principle is as simple as "one person, one euro".
} 
this egalitarian goal will be met perfectly if and only if the nucleolus for the representatives coincides with the population shares. It is not clear, however, how this principle ought to be operationalized in practice. Although it seems straightforward to allocate weights proportional to population sizes, there are often stark discrepancies between voting weight and voting power as illustrated in the beginning of the introduction. We are confronted with a combinatorial second best optimization problem. Second best, because perfect equality is unattainable in general, thus we need to evaluate the social loss associated to deviations from equality. Combinatorial, because there is a finite number of possible games. In that respect the terminology "optimal weights" can be misleading as what matters is the simple game induced by the weights (if there are only three countries, weights are almost meaningless). In addition to that, there is no reason to infer that the optimal simple game will be a weighted majority game ${ }^{11}$. The combinatorial problem is difficult. We introduce a methodology based on variance minimization ${ }^{12}$ for the design of the voting rule and illustrate its application when the number of EU members was relatively small; even in this case, implementing the method is far from easy and we restrict our search to a subset of all simple games ${ }^{13}$. We conclude that the allocation was not fair ${ }^{14}$. In particular, we show that in the Council of Ministers in 1958, Germany got too little weight as compared to France and Italy, and that, surprisingly, the choice to make Luxembourg a dummy was optimal.

The rest of the paper is organized as follows. Section 2 provides a review of the closely related literature. Section 3 describes the first five configurations of the Council of Ministers between 1958 and 1995 which operated under weighted voting rules. We provide the values for the nucleolus and compare them with alternative indices. In section 4 , we repeat the exercise for the qualified voting rules for 15 and 27 members as prescribed by the Treaty of Nice. Section 5 is devoted to the design of the optimal (fair) voting rules. Section 6 deals with the calculation of the optimal voting rule for the earlier stages of the EU. An appendix is dedicated to an overview of the notions from cooperative game theory which are used in this paper, as well as some results on the combinatorics of simple games with special attention to the issue of representation by weights.

\section{Two "New" Power Indices}

A measure of power is a map $\xi$ from the set of simple games $(N, \mathcal{W})$ to the set of $n$-tuples of real numbers. The value $\xi_{i}=\xi_{i}(N, \mathcal{W})$ is the power of player $i$ in the game $(N, \mathcal{W})$, and it satisfies $0 \leq \xi_{i} \leq 1$. The most famous power measures used in the literature are the Banzhaf $(B Z)$ and the Shapley-Shubik $(S S)$ indices. The latter is considered the appropriate power

\footnotetext{
${ }^{11}$ In the utilitarian framework, Barberà and Jackson (2006) show that the optimal voting rule is almost a weighted voting rule.

${ }^{12}$ Other inequality indices like the Gini index or the Kolm-Atkinson indices could be used instead. The results are very similar if the Gini index is used (see p. 31).

${ }^{13}$ Because the optimal second best game is not guaranteed to be a member of the chosen class of games, the problem becomes a third best optimization problem.

${ }^{14}$ The smaller countries have not been systematically overrepresented according to the nucleolus. In particular, the total payoff is divided among the four largest countries in the 1973 and 1981 Councils.
} 
measure in distributive situations (see Felsenthal and Machover (1998) and Laruelle and Valenciano (2008)). Its justification is mainly axiomatic (Shapley, 1953; Dubey, 1975).

In this paper, we introduce two new measures of power which are not derived from any set of axioms but instead as vectors of equilibrium payoffs of positive models of politics. We are interested in the power of a country to approve as well as its power to block a decision. The Banzhaf and the Shapley-Shubik indices give the same answer in both situations ${ }^{15}$. The two new measures may assign different values to the power to approve and to the power to block a proposal by a country.

\subsection{Lobbying and Power : The Nucleolus}

In this section, we show that the nucleolus and more generally, the vectors belonging to the least core of the simple game arise as the vectors of equilibrium payoffs of a game describing the competition between two lobbies to buy the vote of the legislators. This game is due to Groseclose and Snyder (1996) and further analyzed by Banks (2000) and Diermeier and Myerson (1999). Lobbies make offers to the legislators sequentially, and each legislator votes for the lobby that paid him the most.

Le Breton and Zaporozhets (2010) and Le Breton, Sudhölter and Zaporozhets (2010) show that, if lobbying takes place in equilibrium, the set of equilibrium offers coincides with the least core of the cooperative game. The public policy can be biased toward one side or the other depending upon the strength of each lobby and one key parameter called the hurdle factor of the simple game. Le Breton and Zaporozhets (2010) and Le Breton, Sudhölter and Zaporozhets (2010) show how to calculate the hurdle factor. Young (1978 a, b) had already developed a similar model assuming that lobbies move simultaneously and showed that the set of equilibrium offers coincides with the least core if one lobby is much stronger than the other.

As emphasized by Young, the nucleolus $N U(N, \mathcal{W})$ of the simple game $(N, \mathcal{W})$ can be interpreted as the vector of relative prices of the legislators' votes that a lobby has to pay to impose its most preferred outcome in the presence of the opposition. If the first mover favors the status quo, it needs to forestall all possible attempts by the second mover to bribe a winning coalition. The optimal prices are the solutions to the following linear program (the budget of the second mover is normalized to 1 ):

$$
\begin{array}{ll}
\min & \sum_{i \in N} t_{i} \\
\text { s.t. } & \sum_{i \in S} t_{i} \geq 1 \text { for all } S \in \mathcal{W} . \\
& t_{i} \geq 0 \text { for all } i \in N
\end{array}
$$

\footnotetext{
${ }^{15}$ The (absolute) Banzhaf measure of a game coincides with the Banzhaf measure of the dual game in which the blocking coalitions of the original game are winning. There are two other measures, the Coleman (1971) measures, which refer to the probability of being pivotal conditional on the final decision being positive or negative. The two Coleman measures are proportional to the Banzhaf measure and are mutually dual (see Felsenthal and Machover, p. 49).
} 
The solution to this program (rescaled so that total payoffs add up to 1) is the least core of the corresponding cooperative game. It may contain multiple solutions, but the nucleolus is always one of them.

It is important to point out that the set of equilibrium prices crucially depends on the order of moves. If the second mover favors the status quo, it only needs to convince a blocking coalition rather than a winning coalition, and the first mover needs to make sure that no blocking coalition is affordable to the second mover. The corresponding vectors of prices are the solutions to the following linear program, where $\mathcal{B}$ is the set of blocking coalitions:

$$
\begin{array}{ll}
\min & \sum_{i \in N} t_{i} \\
\text { s.t. } & \sum_{i \in S} t_{i} \geq 1 \text { for all } S \in \mathcal{B} . \\
& t_{i} \geq 0 \text { for all } i \in N
\end{array}
$$

The measures of power advocated by Banzhaf and Shapley-Shubik are invariant to the duality operation, i.e. $B Z(N, \mathcal{W})=B Z(N, \mathcal{B})$ and $S S(N, \mathcal{W})=S S(N, \mathcal{B})$. In contrast, $N U(N, \mathcal{W}) \neq N U(N, \mathcal{B})$ except in the case where $(N, \mathcal{W})$ is constant sum.

\subsection{Bargaining and Power : The Nucleolus (Again)}

In this section, we describe the power of the players as their expected equilibrium payoff in a legislative bargaining game introduced by Baron and Ferejohn (1989). The voting rule is represented by a simple voting game $(N, \mathcal{W})$.

Bargaining proceeds as follows. At every round $t=1,2, \ldots$ Nature selects a random proposer: player $i$ is selected with probability $p_{i}$. This player proposes a distribution of the budget $\left(x_{1}, \ldots, x_{n}\right)$ with $x_{j} \geq 0$ for all $j=1, \ldots n$ and $\sum_{j=1}^{n} x_{j}=1$. The proposal is voted upon immediately (closed rule). If the coalition of voters in favor of the proposal is winning, the proposal is implemented and the game ends; otherwise the game proceeds to the next period in which Nature selects a new proposer. Players are risk neutral and discount future payoffs by a factor $\delta_{i} \in[0,1]$. A (pure) strategy for player $i$ is a sequence $\sigma_{i}=\left(\sigma_{i}^{t}\right)_{t=1}^{\infty}$, where $\sigma_{i}^{t}($.$) , the t$ th round strategy of player $i$, prescribes a proposal $x$ and a response to all possible proposals by the other players. Players may condition their actions on the history of play; however we will focus on equilibria in which they do not.

The solution concept is stationary subgame perfect equilibrium (SSPE). Stationarity requires that players follow the same strategy at every round $t$ regardless of past offers and responses to past offers. Banks and Duggan (2000) have shown that an $\mathrm{SSPE}^{16}$ always exists

\footnotetext{
${ }^{16}$ The main predictions of the model in the absence of veto players are the following. First, there is immediate agreement. Even without discounting there is a pressure to reach agreement in the first period because of the risk of being excluded afterward. Second, all coalition partners with a positive expected payoff must be pivotal, since otherwise it would be a waste of resources for the proposer. Third, the proposer receives a disproportionate share of the pie, because he always buys the cheapest coalition and pays its members just enough to secure the acceptance of the proposal.
} 
in this type of bargaining model. In addition, Eraslan and McLennan (2006) have shown that all SSPE lead to the same expected equilibrium payoffs ${ }^{17}$.

In the case where $p_{i}=\frac{1}{n}$ and $\delta_{i}=1$ for all $i=1, \ldots, n$, we denote by $B F(N, \mathcal{W})$ the unique vector of equilibrium payoffs attached to the SSPE of the bargaining game. Hereafter, we will refer to this vector as the Baron-Ferejohn measure of power attached to the simple game $(N, \mathcal{W})$.

Montero (2006) has analyzed the above bargaining game in the case where $\delta_{i}=\delta$ for all $i=1, \ldots, n$. She shows that if the vector $p$ coincides with the nucleolus, then $p$ is the unique vector of equilibrium payoffs. In her terminology, the nucleolus is a self-confirming measure of power. She also shows that in equilibrium all players are equally likely to be in the coalition that forms (except for players who get 0 according to the nucleolus, who may appear more infrequently). This is consistent with an interpretation of the nucleolus as a system of competitive prices: if players are paid a competitive price, there is no reason for some coalition partners to be more desirable than others (see Montero (2005), section 3).

It is worth noting that Kalandrakis (2006) shows that any vector from the simplex is the vector of equilibrium payoffs of the Baron-Ferejohn game for some choice of the recognition probabilities. In particular, the Shapley-Shubik index can be obtained in this way. However, the nucleolus is much more robust to changes in the recognition probabilities. For example, in the weighted majority game $[3 ; 2,1,1,1]$ if we assume $p_{2}=p_{3}=p_{4}$, it turns out that the nucleolus is the equilibrium payoff for $0<p_{1} \leq 0.5$ (a broad interval encompassing equal probabilities for all players and probabilities proportional to votes) whereas the ShapleyShubik index arises only if $p_{1}=3 / 5$ (Montero (2006), example 9).

\section{Five Voting Bodies: Descriptive Analysis of Power}

This section is purely descriptive. We analyze five weighted majority voting games associated to the Council of Ministers of the European Union in 1958, 1973, 1981, 1986 and 1995 (Table 1 is adapted from Felsenthal and Machover, 2001), and compare the distribution of the decision power according to the four different power measures.

We provide values for the Banzhaf (BZ) and the Shapley-Shubik (SS) indices (calculated using the webpage of D. Leech), the nucleolus (denoted by NU and calculated using a Maple program based on Matsui and Matsui (2000)), the nucleolus of the dual game (denoted NU(b)) and two indices obtained from the Baron-Ferejohn noncooperative game assuming equal recognition probabilities. Expected payoffs for the original Baron-Ferejohn game are denoted by BF and taken from Montero (2007); some of these results also appear in Snyder et al. $(2005)^{18}$. Expected payoffs in the Baron-Ferejohn model with respect to the dual game $(N, \mathcal{B})$ are calculated below and denoted by $\mathbf{B F}(\mathbf{b})$. Besides being relevant to lobbying, the dual game can be relevant to bargaining if the first coalition that forms is able to set the status quo and commit itself to vote against any alternative proposal.

\footnotetext{
${ }^{17}$ Special cases of this result were proven in Eraslan (2002), Montero (2006) and Yan (2009).

${ }^{18}$ Montero (2007) computes expected payoffs for the 1958, 1973 and 1981 Councils. Snyder et al.'s (2005) table 2 contains expected payoffs for 1958 and 1973. The calculations coincide for 1958 but disagree for 1973.
} 
Table 1: Weights and quota in the Council of Ministers.

\begin{tabular}{llllll}
\hline \hline Country & $\mathbf{1 9 5 8}$ & $\mathbf{1 9 7 3}$ & $\mathbf{1 9 8 1}$ & $\mathbf{1 9 8 6}$ & $\mathbf{1 9 9 5}$ \\
\hline Germany & 4 & 10 & 10 & 10 & 10 \\
Italy & 4 & 10 & 10 & 10 & 10 \\
France & 4 & 10 & 10 & 10 & 10 \\
UK & - & 10 & 10 & 10 & 10 \\
Spain & - & - & - & 8 & 8 \\
Belgium & 2 & 5 & 5 & 5 & 5 \\
Netherlands & 2 & 5 & 5 & 5 & 5 \\
Greece & - & - & 5 & 5 & 5 \\
Portugal & - & - & - & 5 & 5 \\
Sweden & - & - & - & - & 4 \\
Austria & - & - & - & - & 4 \\
Denmark & - & 3 & 3 & 3 & 3 \\
Ireland & - & 3 & 3 & 3 & 3 \\
Finland & - & - & - & - & 3 \\
Luxembourg & 1 & 2 & 2 & 2 & 2 \\
\hline Quota & 12 & 41 & 45 & 54 & 62 \\
Total votes & 17 & 58 & 63 & 76 & 87 \\
Quota (\%) & 70.59 & 70.69 & 71.43 & 71.05 & 71.26 \\
\hline
\end{tabular}

\subsection{Power Distribution in 1958}

The European Community is represented by the weighted majority game $[12 ; 4,4,4,2,2,1]$. As one can easily see Luxembourg is not in any minimal winning coalition, and the game can be equivalently represented as $[6 ; 2,2,2,1,1,0]$.

First, we look at the expected equilibrium payoffs in the bargaining game with equal probabilities of being a proposer and focusing on blocking coalitions. Denote by $x, y$ and $z$ respectively the expected payoffs for players of type 2,1 and 0 . We conjecture that $x=y$ in equilibrium. If this is the case, the equilibrium strategies might be summarized as follows:

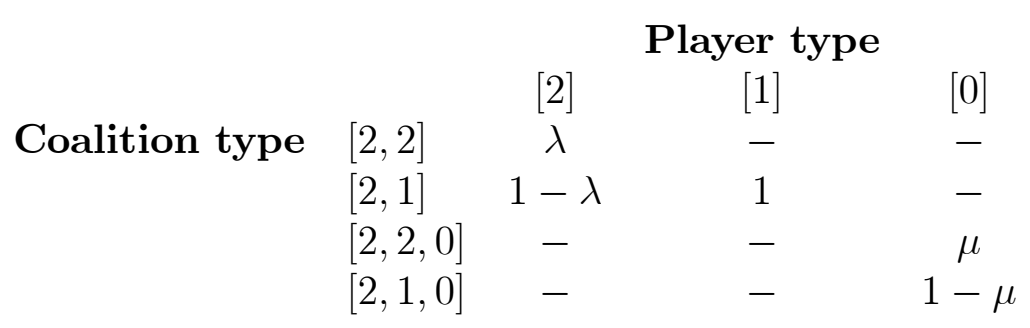

In the table we indicate the probability of proposing each coalition type by each player 
type. ${ }^{19}$ The equations for the players' expected payoffs are:

$$
\begin{aligned}
x & =\frac{1}{6}(1-x)+\frac{2}{6} \frac{\lambda}{2} x+\frac{2}{6} \frac{1}{3} x+\frac{1}{6}\left(\frac{2}{3} \mu+\frac{1-\mu}{3}\right) x \\
y & =\frac{1}{6}(1-x)+\frac{3}{6} \frac{1-\lambda}{2} y+\frac{1}{6} \frac{1-\mu}{2} y \\
z & =\frac{1}{6}(1-2 x) \\
x & =y
\end{aligned}
$$

The solution is: $0 \leq \mu \leq 1, \lambda=\frac{6-5 \mu}{15}, x=y=\frac{5}{28} \approx 0.179, z=\frac{3}{28} \approx 0.107$. The values found for the probabilities are between 0 and 1 and no player can gain by proposing an alternative coalition given the values of $x, y$ and $z$; hence we have an equilibrium.

Interestingly, the medium-size countries get the same payoff as the large ones, and the small country gets a disproportionately high payoff as well. Part of the reason is that the small and medium countries have a disproportionately high proposal power: the probability of being selected as a proposer is the same for all the countries and equals $1 / 6$. Note also that Luxembourg is a dummy but gets a positive expected payoff because it is allowed to make proposals.

Denote by $x, y$ and $z$ respectively the payoffs for players of type 2,1 and 0 . In order to calculate the nucleolus (NU) we solve the problem (1) which looks like

$$
\begin{array}{cl}
\min & 3 x+2 y \\
\text { s.t. } & 2 x+2 y \geq 1 \\
& 3 x \geq 1 \\
& x, y \geq 0
\end{array}
$$

The solution of this problem is $x=\frac{1}{3}$ and $y=\frac{1}{6}$, and the value of the program (the hurdle factor) is $\gamma=1.333$. A player of type 2 receives twice as much as a player of type 1 , which is intuitive since it can be replaced by two players of type 1 in a minimal winning coalition. This substitutability property often holds for the nucleolus in contrast to other power indices, but it does not hold in all cases. In particular, it does not hold for 1973 and 1981 as we will see below.

If we look at the dual game, the nucleolus is the solution (up to a normalization) of the following program:

$$
\begin{gathered}
\min 3 x+2 y \\
\text { s.t. } x+y \geq 1 \\
2 x \geq 1 \\
x, y \geq 0
\end{gathered}
$$

\footnotetext{
${ }^{19}$ For example, a player of type [2] proposes a coalition of type [2,2] with probability $\lambda$. Because each proposer of type [2] belongs to two coalitions of type [2,2], each of them is proposed with probability $\lambda / 2$. A type [0] player proposes a coalition of type $[2,1,0]$ with probability $1-\mu$. There are 6 such coalitions, and each type [1] player belongs to 3 of them. Thus, if type [0] is selected to be the proposer, each type [1] player receives a proposal with probability $(1-\mu) / 2$.
} 
The solution now is $\left(\frac{1}{2}, \frac{1}{2}\right)$ and the value of the program (the dual hurdle factor) is $\gamma=2.5$. Note that players of types 2 and 1 get the same payoff according to the nucleolus even though they are not interchangeable.

The power measures are summarized in Table 2 :

Table 2: Power distribution in 1958.

\begin{tabular}{lllllll}
\hline \hline Country & SS & BZ & BF & BF (b) & $\begin{array}{l}\text { NU } \\
\gamma=1.333\end{array}$ & $\begin{array}{l}\text { NU(b) } \\
\gamma=2.5\end{array}$ \\
\hline Germany & 0.233 & 0.238 & 0.238 & 0.179 & 0.250 & 0.200 \\
Italy & 0.233 & 0.238 & 0.238 & 0.179 & 0.250 & 0.200 \\
France & 0.233 & 0.238 & 0.238 & 0.179 & 0.250 & 0.200 \\
Netherlands & 0.150 & 0.143 & 0.119 & 0.179 & 0.125 & 0.200 \\
Belgium & 0.150 & 0.143 & 0.119 & 0.179 & 0.125 & 0.200 \\
Luxembourg & 0 & 0 & 0.048 & 0.107 & 0 & 0 \\
\hline
\end{tabular}

\subsection{Power Distribution in 1973}

The voting body is represented by the weighted majority game $[41 ; 10,10,10,10,5,5,3,3,2]$.

Again, we are looking for the expected equilibrium payoffs in the dual game, and we denote by $x, y, z$ and $w$ the expected payoffs for players of type 10, 5, 3 and 2 respectively. We look for an equilibrium in which $y=z$ and $x<2 y$. If such an equilibrium exists, [10,10] is the cheapest coalition type for proposer type [10], and $[10,5,3]$ is the cheapest coalition type for [3]. Type [5] is indifferent between $[10,5,5]$ and $[10,5,3]$; we will look for an equilibrium in which $[10,5,3]$ is proposed with certainty. Type [2]'s cheapest minimal winning coalition is $[10,3,3,2]$, but this is not the optimal coalition for this type because $[10,10,2]$ is cheaper. The equilibrium strategies might be summarized as follows:

\begin{tabular}{clcccc} 
& \multicolumn{5}{c}{ Player type } \\
Coalition type & {$[10,10]$} & $1(3)$ & {$[5]$} & {$[3]$} & {$[2]$} \\
& {$[10,5,3]$} & - & $1(8)$ & $1(8)$ & - \\
& {$[10,10,2]$} & - & - & - & $1(4)$
\end{tabular}


The equations for the expected payoffs are given by:

$$
\begin{aligned}
x & =\frac{1}{9}(1-x)+\frac{3}{9} \frac{1}{3} x+\frac{4}{9} \frac{1}{4} x+\frac{1}{9} \frac{1}{2} x \\
y & =\frac{1}{9}(1-x-z)+\frac{2}{9} \frac{1}{2} y \\
z & =\frac{1}{9}(1-x-y)+\frac{2}{9} \frac{1}{2} z \\
w & =\frac{1}{9}(1-2 x) \\
y & =z
\end{aligned}
$$

Expected equilibrium payoffs are:

$$
x=\frac{2}{15} \approx 0.133, y=z=\frac{13}{135} \approx 0.096, w=\frac{11}{135} \approx 0.081
$$

Because the expected payoffs we found satisfy our original conjecture $x<2 y$, we have found an equilibrium. Surprisingly, expected payoffs for the smaller countries are quite large and do not differ much between player types.

To calculate the nucleolus we solve the linear program:

$$
\begin{array}{ll}
\min & 4 x+2 y+2 z+w \\
\text { s.t. } & 4 x+y \geq 1 \\
& 4 x+z \geq 1 \\
& 4 x+w \geq 1 \\
& 3 x+2 y+z \geq 1 \\
& 3 x+2 y+w \geq 1 \\
& 3 x+y+2 z \geq 1 \\
& x, y, z, w \geq 0
\end{array}
$$

The solution is $(1 / 3,0,0,0)$ and the value of the program is $4 / 3$. As compared to 1958 the hurdle factor does not change, as well as the power of the big countries. However, other countries, even though they are not dummies, get zero. Giving 0 to nondummies is impossible for SS and BZ but is not unusual for the nucleolus; as a result the nucleolus is very different from other indices in this example.

Looking at the minimal blocking coalitions we need to solve:

$$
\begin{array}{ll}
\min & 4 x+2 y+2 z+w \\
\text { s.t. } & x+y+z \geq 1 \\
& x+2 y \geq 1 \\
2 x & \geq 1 \\
2 y & +2 z+w \geq 1 \\
& x+2 z+w \geq 1 \\
& x, y, z, w \geq 0
\end{array}
$$


The solution is $\left(\frac{1}{6}, \frac{1}{12}, \frac{1}{12}, 0\right)$ and the dual hurdle factor is $\gamma=3$. Even though Luxembourg is not a dummy anymore it still gets 0 . The dual hurdle factor is greater than in the previous case, which means that the Council became less vulnerable to lobbying.

The power measures are summarized in Table 3.

Table 3: Power distribution in 1973.

\begin{tabular}{lllllll}
\hline \hline Country & SS & BZ & BF & BF (b) & $\begin{array}{l}\text { NU } \\
\gamma=1.333\end{array}$ & $\begin{array}{l}\text { NU (b) } \\
\gamma=3.0\end{array}$ \\
\hline Germany & 0.179 & 0.167 & 0.159 & 0.133 & 0.250 & 0.167 \\
Italy & 0.179 & 0.167 & 0.159 & 0.133 & 0.250 & 0.167 \\
France & 0.179 & 0.167 & 0.159 & 0.133 & 0.250 & 0.167 \\
UK & 0.179 & 0.167 & 0.159 & 0.133 & 0.250 & 0.167 \\
Belgium & 0.081 & 0.091 & 0.079 & 0.096 & 0 & 0.083 \\
Netherlands & 0.081 & 0.091 & 0.079 & 0.096 & 0 & 0.083 \\
Denmark & 0.057 & 0.066 & 0.071 & 0.096 & 0 & 0.083 \\
Ireland & 0.057 & 0.066 & 0.071 & 0.096 & 0 & 0.083 \\
Luxembourg & 0.001 & 0.016 & 0.063 & 0.081 & 0 & 0 \\
\hline
\end{tabular}

\subsection{Power Distribution in 1981}

As pointed out in Montero (2007) the representation [45; 10, 10, 10, 10, 5, 5, 5, 3, 3,2] is equivalent to $[18 ; 4,4,4,4,2,2,2,1,1,1]$. The dual game is then $[8 ; 4,4,4,4,2,2,2,1,1,1]$.

The nucleolus is the solution of the linear program:

$$
\begin{array}{ll}
\min & 4 x+3 y+3 z \\
\text { s.t. } & 4 x+y \geq 1 \\
& 4 x+2 z \geq 1 \\
& 3 x+3 y \geq 1 \\
& 3 x+2 y+2 z \geq 1 \\
& x, y, z \geq 0
\end{array}
$$

The minimum is reached at $(1 / 3,0,0)$, and the value of this minimum is $4 / 3$. In fact, nothing is changed as compared to 1973.

The following linear program: 


$$
\begin{array}{cl}
\min & 4 x+3 y+3 z \\
\text { s.t. } & 2 x \geq 1 \\
& x+2 y \geq 1 \\
& x+y+2 z \geq 1 \\
3 y & +2 z \geq 1 \\
x, y, z \geq 0 &
\end{array}
$$

gives the solution if we are interested in blocking coalitions. The nucleolus in this case is $(0.16,0.08,0.04)$ and the dual hurdle factor is $\gamma=3.125$.

When calculating expected payoffs in the dual game, we look for an equilibrium in which $x<2 y, y<2 z$ and $x<y+z$ (the latter inequality implies that [4,4,1] is the optimal coalition type for player type [1]). Then, the optimal strategies can be summarized in the following table:

\begin{tabular}{llccc} 
& & \multicolumn{3}{c}{ Player type } \\
Coalition type & & {$[4]$} & {$[2]$} & {$[1]$} \\
& {$[4,4]$} & $1(3)$ & - & - \\
& {$[4,2,2]$} & - & $1(8)$ & - \\
& {$[4,4,1]$} & - & - & $1(6)$
\end{tabular}

The system for the equilibrium expected payoffs is:

$$
\begin{aligned}
x & =\frac{1}{10}(1-x)+\frac{3}{10} \frac{1}{3} x+\frac{3}{10} \frac{2}{8} x+\frac{3}{10} \frac{3}{6} x \\
y & =\frac{1}{10}(1-x-y)+\frac{2}{10} \frac{4}{8} y \\
z & =\frac{1}{10}(1-2 x)
\end{aligned}
$$

The solution is:

$$
x=\frac{4}{31} \approx 0.129, y=\frac{27}{310} \approx 0.087 \text { and } z=\frac{23}{310} \approx 0.074
$$

Because the values of $x, y$ and $z$ we found satisfy our original assumptions of $x<2 y$, $y<2 z$ and $x<y+z$, we have found an equilibrium.

The power measures are summarized in Table 4.

\subsection{Power Distribution in 1986}

The game is described as $[54 ; 10,10,10,10,8,5,5,5,5,3,3,2]$. The dual game can be written as $[23 ; 10,10,10,10,8,5,5,5,5,3,3,2]$. By $\omega$ we denote the number of minimal winning coalitions. Table 5 summarizes the power measures for this voting rule. Unlike in the previous two cases, the four power measures are not too different. Some groups of equivalent players 
Table 4: Power distribution in 1981.

\begin{tabular}{lllllll}
\hline \hline Country & SS & BZ & BF & BF (b) & $\begin{array}{l}\text { NU } \\
\gamma=1.333\end{array}$ & $\begin{array}{l}\text { NU (b) } \\
\gamma=3.125\end{array}$ \\
\hline Germany & 0.174 & 0.158 & 0.160 & 0.129 & 0.250 & 0.160 \\
Italy & 0.174 & 0.158 & 0.160 & 0.129 & 0.250 & 0.160 \\
France & 0.174 & 0.158 & 0.160 & 0.129 & 0.250 & 0.160 \\
UK & 0.174 & 0.158 & 0.160 & 0.129 & 0.250 & 0.160 \\
Belgium & 0.071 & 0.082 & 0.080 & 0.087 & 0 & 0.080 \\
Netherlands & 0.071 & 0.082 & 0.080 & 0.087 & 0 & 0.080 \\
Greece & 0.071 & 0.082 & 0.080 & 0.087 & 0 & 0.080 \\
Denmark & 0.030 & 0.041 & 0.040 & 0.074 & 0 & 0.040 \\
Ireland & 0.030 & 0.041 & 0.040 & 0.074 & 0 & 0.040 \\
Luxembourg & 0.030 & 0.041 & 0.040 & 0.074 & 0 & 0.040 \\
\hline
\end{tabular}

get the same payoff according to the nucleolus: a player with 10 votes gets the same payoff as two players with 5 votes, and the player with 8 votes gets the same as two players with 5 and 3 votes. Nevertheless, Luxembourg still gets 0 even though it can replace a large country together with Spain.

Table 5: Power distribution in 1986.

\begin{tabular}{|c|c|c|c|c|}
\hline Country & 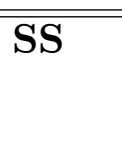 & $\overline{\mathrm{BZ}}$ & $\begin{array}{l}\mathbf{N U} \\
\gamma=1.38 \\
\omega=135\end{array}$ & $\begin{array}{l}\text { NU (b) } \\
\gamma=3.2 \\
\omega=182\end{array}$ \\
\hline Germany & 0.134 & 0.129 & 0.138 & 0.125 \\
\hline Italy & 0.134 & 0.129 & 0.138 & 0.125 \\
\hline France & 0.134 & 0.129 & 0.138 & 0.125 \\
\hline UK & 0.134 & 0.129 & 0.138 & 0.125 \\
\hline Spain & 0.111 & 0.109 & 0.103 & 0.125 \\
\hline Belgium & 0.064 & 0.067 & 0.069 & 0.063 \\
\hline Netherlands & 0.064 & 0.067 & 0.069 & 0.063 \\
\hline Greece & 0.064 & 0.067 & 0.069 & 0.063 \\
\hline Portugal & 0.064 & 0.067 & 0.069 & 0.063 \\
\hline Denmark & 0.043 & 0.046 & 0.034 & 0.063 \\
\hline Ireland & 0.043 & 0.046 & 0.034 & 0.063 \\
\hline Luxembourg & 0.012 & 0.018 & 0 & 0 \\
\hline
\end{tabular}




\subsection{Power Distribution in 1995}

The game is described as $[62 ; 10,10,10,10,8,5,5,5,5,4,4,3,3,3,2]$ with total weight 87 . The dual game is $[26 ; 10,10,10,10,8,5,5,5,5,4,4,3,3,3,2]$. Table 6 summarizes the power measures for this voting rule. It is interesting to note that this is the only case in which the nucleolus is proportional to the weights.

Table 6: Power distribution in $\mathbf{1 9 9 5 .}$

\begin{tabular}{lllll}
\hline \hline Country & SS & BZ & $\begin{array}{l}\text { NU } \\
\gamma=1.4\end{array}$ & $\begin{array}{l}\text { NU (b) } \\
\gamma=3.33 \\
\end{array}$ \\
& & & $\omega=829$ & $\omega=1270$ \\
\hline Germany & 0.117 & 0.112 & 0.115 & 0.1 \\
Italy & 0.117 & 0.112 & 0.115 & 0.1 \\
France & 0.117 & 0.112 & 0.115 & 0.1 \\
UK & 0.117 & 0.112 & 0.115 & 0.1 \\
Spain & 0.095 & 0.092 & 0.092 & 0.1 \\
Belgium & 0.055 & 0.059 & 0.057 & 0.05 \\
Netherlands & 0.055 & 0.059 & 0.057 & 0.05 \\
Greece & 0.055 & 0.059 & 0.057 & 0.05 \\
Portugal & 0.055 & 0.059 & 0.057 & 0.05 \\
Sweden & 0.045 & 0.048 & 0.046 & 0.05 \\
Austria & 0.045 & 0.048 & 0.046 & 0.05 \\
Denmark & 0.035 & 0.036 & 0.034 & 0.05 \\
Ireland & 0.035 & 0.036 & 0.034 & 0.05 \\
Finland & 0.035 & 0.036 & 0.034 & 0.05 \\
Luxembourg & 0.021 & 0.023 & 0.023 & 0.05 \\
\hline
\end{tabular}

\section{Qualified Majority Voting in the Treaty of Nice}

\subsection{QMV in non-enlarged CM}

The Treaty of Nice changed the votes of the member states and the quota to $\mathcal{W}_{15}=$ $[169 ; 29,29,29,29,27,13,12,12,12,10,10,7,7,7,4]$. It also introduced the additional requirement that the member states constituting the qualified majority represent at least $62 \%$ of the total population. A majority of member states is also mentioned, but this turns out to be redundant (see Felsenthal and Machover (2001)).

The rule $\mathcal{P}_{15}=[2327 ; 820,592,590,576,394,158,105,102,100,89,81,53,52,37,4]$ (total weight is 3753 ) is the weighted rule whose weights are population sizes of 15 countries and 
quota is $62 \%$. The following Table 7 presents the results of computing the nucleolus for $\mathcal{W}_{15}$ as well as for the double majority system $\mathcal{W}_{15} \cap \mathcal{P}_{15}$.

Table 7: Power distribution for the 15 EU countries under the nucleolus.

\begin{tabular}{lllll}
\hline \hline Country & \multicolumn{2}{c}{$\mathcal{W}_{15}$} & \multicolumn{2}{c}{$\mathcal{W}_{15} \cap \mathcal{P}_{15}$} \\
& NU & NU(b) & NU & NU(b) \\
& $\gamma=1.4$ & $\gamma=3.414$ & $\gamma=1.4$ & $\gamma=3.483$ \\
& $\omega=775$ & $\omega=1018$ & $\omega=760$ & $\omega=1490$ \\
\hline Germany & 0.122 & 0.121 & 0.122 & 0.139 \\
Italy & 0.122 & 0.121 & 0.122 & 0.119 \\
France & 0.122 & 0.121 & 0.122 & 0.119 \\
UK & 0.122 & 0.121 & 0.122 & 0.119 \\
Spain & 0.112 & 0.111 & 0.112 & 0.109 \\
Belgium & 0.051 & 0.061 & 0.051 & 0.059 \\
Netherlands & 0.051 & 0.051 & 0.051 & 0.050 \\
Greece & 0.051 & 0.051 & 0.051 & 0.050 \\
Portugal & 0.051 & 0.051 & 0.051 & 0.050 \\
Sweden & 0.041 & 0.040 & 0.041 & 0.050 \\
Austria & 0.041 & 0.040 & 0.041 & 0.040 \\
Denmark & 0.031 & 0.030 & 0.031 & 0.030 \\
Ireland & 0.031 & 0.030 & 0.031 & 0.030 \\
Finland & 0.031 & 0.030 & 0.031 & 0.030 \\
Luxembourg & 0.020 & 0.020 & 0.020 & 0.020 \\
\hline
\end{tabular}

\subsection{QMV in a 27-member CM}

Following Felsenthal and Machover (2001) and Bilbao et al. (2002) we consider different variants involving votes, population and/or number of member countries.

The first variant is a double majority system $v_{1} \cap v_{2}$, or $v_{1} \cap v_{3}$. The rule $v_{1}$ is the weighted rule with votes described by

$\mathcal{W}_{27}=[255 ; 29,29,29,29,27,27,14,13,12,12,12,12,12,10,10,10,7,7,7,7,7,4,4,4,4,4,3]$.

The rule $v_{2}$ is rule $\mathcal{P}_{27}$, the weighted rule whose weights are population shares (per thousand) of the 27 members and whose quota is equal to $62 \%$ :

$\mathcal{P}_{27}=[620 ; 170,123,122,120,82,80,47,33,22,21,21,21,21,18,17,17,11,11,11,8,8,5,4,3,2,1,1]$.

Finally, $v_{3}$ is $\mathcal{M}_{27}$, the ordinary majority rule:

$\mathcal{M}_{27}=[14 ; 1,1,1,1,1,1,1,1,1,1,1,1,1,1,1,1,1,1,1,1,1,1,1,1,1,1,1]$.

The second variant is a triple majority system of votes, population and member countries $v_{1} \cap v_{2} \cap v_{3}$, where $v_{1}, v_{2}$ and $v_{3}$ are as before.

We report the number of minimal winning coalitions for each rule below. 
Table 8: Minimal winning coalitions for the 27 EU countries under different rules.

\begin{tabular}{ll}
\hline \hline rule & $\omega$ \\
\hline $\mathcal{W}_{27}$ & 561645 \\
$\mathcal{W}_{27} \cap \mathcal{P}_{27}$ & 561642 \\
$\mathcal{W}_{27} \cap \mathcal{M}_{27}$ & 561823 \\
$\mathcal{W}_{27} \cap \mathcal{M}_{27} \cap \mathcal{P}_{27}$ & 561820 \\
\hline
\end{tabular}

Felsenthal and Machover (2001) show that $\mathcal{W}_{27}$ and $\mathcal{W}_{27} \cap \mathcal{M}_{27} \cap \mathcal{P}_{27}$ differ in only 23 coalitions (out of the 2,718,774 winning coalitions of $\mathcal{W}_{27}, 23$ become losing in $\mathcal{W}_{27} \cap \mathcal{M}_{27} \cap$ $\mathcal{P}_{27}$ ); this difference has a negligible impact on the Banzhaf index. Similarly, we observe little difference between the four rules in terms of the number of minimal winning coalitions ${ }^{20}$. The hurdle factor $\gamma$ is not affected by the additional requirements and remains the same $(\gamma=1.353)$ for all rules. Likewise, the nucleolus assigns the same values to the countries under the four rules (see table 9), and these values are proportional to the weights in $\mathcal{W}_{27}$.

\footnotetext{
${ }^{20}$ Note that while the number of winning coalitions cannot increase under intersection, the number of minimal winning coalitions may increase or decrease.
} 
Table 9: Power distribution for the 27 EU countries according to the nucleolus under any of the considered rules.

\begin{tabular}{ll}
\hline \hline Country & NU \\
\hline Germany & 0.084 \\
UK & 0.084 \\
France & 0.084 \\
Italy & 0.084 \\
Spain & 0.078 \\
Poland & 0.078 \\
Romania & 0.041 \\
Netherlands & 0.038 \\
Greece & 0.035 \\
Czech Republic & 0.035 \\
Belgium & 0.035 \\
Hungary & 0.035 \\
Portugal & 0.035 \\
Sweden & 0.029 \\
Bulgaria & 0.029 \\
Austria & 0.029 \\
Slovak Republic & 0.020 \\
Denmark & 0.020 \\
Finland & 0.020 \\
Ireland & 0.020 \\
Lithuania & 0.020 \\
Latvia & 0.012 \\
Slovenia & 0.012 \\
Estonia & 0.012 \\
Cyprus & 0.012 \\
Luxembourg & 0.012 \\
Malta & 0.009 \\
\hline &
\end{tabular}

\section{The Power of the European Citizens and the Optimal Decision Rule}

In the previous sections we calculated several measures of the power of each nation (representative) in the Council of Ministers of the European Union. In this section, we will focus on the nucleolus and we will adopt a normative perspective. As already explained, focusing on the nucleolus simply means that we are interested in European policy issues which can be described formally as distributive politics. Something has to be shared among the members of the Council of Ministers and ultimately among the European citizens and the nucleolus is the reduced form of equilibrium for several alternative game forms spanning bargaining and 
lobbying. To fix ideas, let us for the time being interpret this pie as the gains (measured in appropriate units) resulting from European coordination. Fairness requires equal division of these gains between all citizens, which implies that each country should receive a share proportional to its population size. If there were no intermediate political bodies i.e. if the simple game was a majority game with the set of all European citizens as the set of players, then all the coordinates of the nucleolus would be equal and proportionality would be fulfilled. Unfortunately, we are in a second best environment: the negotiation takes place across the countries, and only in a second stage is the share obtained by each country divided among the citizens of the country. We need to evaluate the citizens' indirect power via their representatives.

Let the simple voting game $\Gamma=(M, \mathcal{W})$ describe the decision-making process at the council, where $M=\{1, \ldots, m\}$ is the set of countries and $\mathcal{W}$ is set of all winning coalitions. The set of citizens is denoted by $N=N_{1} \cup \ldots \cup N_{m}$, and the number of citizens of country $i$ is denoted by $n_{i}$. Denoting the nucleolus of the game $\Gamma$ as $\nu$, the citizens' payoffs are given by

$$
y=(\underbrace{\frac{\nu_{1}}{n_{1}}, \ldots, \frac{\nu_{1}}{n_{1}}}_{n_{1} \text { times }}, \ldots, \underbrace{\frac{\nu_{m}}{n_{m}}, \ldots, \frac{\nu_{m}}{n_{m}}}_{n_{m} \text { times }}) .
$$

Remark 1 Citizens' indirect powers are equal for all $i \in N$ iff the nucleolus paypoffs of the delegates $\nu_{j}$ are equal to the respective population rates $\frac{n_{j}}{n}$.

The optimization variable is the simple game $(M, \mathcal{W})$. There is a finite number of possible choices. This number can be large if we do not impose any restrictions on the nature of the simple game. In appendix 3 we report some results from the literature on the enumeration of all simple games or important families of simple games. One of the most important classes is that of constant-sum weighted majority games. Peleg (1968) showed that, if we assign zero weight to dummy players, the unique normalized homogeneous representation of a constantsum homogeneous weighted majority game $(N, \mathcal{W})$ coincides with the nucleolus of $(N, v)$. If the game generated by the weights $\omega_{i}=n_{i}$ and the quota $\frac{\sum_{i \in M} \omega_{i}}{2}$ is homogeneous and has no dummy players, then the solution of our problem is trivial as we can get the first best; however this will only happen by coincidence. In what follows, we will formulate the combinatorial optimization problem that we consider and derive the optimal simple game $(M, \mathcal{W})$. Before doing so, it is useful to evaluate the actual choices of $(M, \mathcal{W})$ for the five stages of European enlargement which are considered in this paper. In the following two tables 10 and 11 we show the population ratios taken from Felsenthal and Machover (1998, 2004) and the nucleolus taken from the tables in the previous section. An asterisk indicates an occurrence of the paradox of new members: a member state's relative power has increased although its relative weight has decreased as a result of the accession of the new members. 
Table 10: Population and the nucleolus in the Council of Ministers 1958-1995.

\begin{tabular}{llllllllllll}
\hline \hline Country & \multicolumn{1}{c}{$\mathbf{1 9 5 8}$} & \multicolumn{2}{c}{$\mathbf{1 9 7 3}$} & \multicolumn{2}{c}{$\mathbf{1 9 8 1}$} & \multicolumn{2}{c}{$\mathbf{1 9 8 6}$} & \multicolumn{2}{c}{$\mathbf{1 9 9 5}$} \\
& $\frac{n_{j}}{n}$ & $\mathbf{N U}$ & $\frac{n_{j}}{n}$ & $\mathbf{N U}$ & $\frac{n_{j}}{n}$ & $\mathbf{N U}$ & $\frac{n_{j}}{n}$ & $\mathbf{N U}$ & $\frac{n_{j}}{n}$ & $\mathbf{N U}$ \\
\hline France & 0.266 & $\mathbf{0 . 2 5 0}$ & 0.203 & $\mathbf{0 . 2 5 0}$ & 0.200 & $\mathbf{0 . 2 5 0}$ & 0.172 & $\mathbf{0 . 1 3 8}$ & 0.156 & $\mathbf{0 . 1 1 5}$ \\
Germany & 0.322 & $\mathbf{0 . 2 5 0}$ & 0.242 & $\mathbf{0 . 2 5 0}$ & 0.228 & $\mathbf{0 . 2 5 0}$ & 0.189 & $\mathbf{0 . 1 3 8}$ & 0.220 & $\mathbf{0 . 1 1 5}$ \\
Italy & 0.291 & $\mathbf{0 . 2 5 0}$ & 0.214 & $\mathbf{0 . 2 5 0}$ & 0.209 & $\mathbf{0 . 2 5 0}$ & 0.176 & $\mathbf{0 . 1 3 8}$ & 0.154 & $\mathbf{0 . 1 1 5}$ \\
Belgium & 0.053 & $\mathbf{0 . 1 2 5}$ & 0.038 & $\mathbf{0}$ & 0.036 & $\mathbf{0}$ & 0.031 & $\mathbf{0 . 0 6 9}$ & 0.027 & $\mathbf{0 . 0 5 7}$ \\
Netherlands & 0.066 & $\mathbf{0 . 1 2 5}$ & 0.052 & $\mathbf{0}$ & 0.053 & $\mathbf{0}$ & 0.045 & $\mathbf{0 . 0 6 9}$ & 0.042 & $\mathbf{0 . 0 5 7}$ \\
Luxembourg & 0.002 & $\mathbf{0}$ & 0.001 & $\mathbf{0}$ & 0.001 & $\mathbf{0}$ & 0.001 & $\mathbf{0}$ & 0.001 & $\mathbf{0 . 0 2 3}$ \\
UK & - & - & 0.218 & $\mathbf{0 . 2 5 0}$ & 0.205 & $\mathbf{0 . 2 5 0}$ & 0.176 & $\mathbf{0 . 1 3 8}$ & 0.157 & $\mathbf{0 . 1 1 5}$ \\
Denmark & - & - & 0.019 & $\mathbf{0}$ & 0.019 & $\mathbf{0}$ & 0.016 & $\mathbf{0 . 0 3 4}$ & 0.014 & $\mathbf{0 . 0 3 4}$ \\
Ireland & - & - & 0.012 & $\mathbf{0}$ & 0.013 & $\mathbf{0}$ & 0.011 & $\mathbf{0 . 0 3 4}$ & 0.010 & $\mathbf{0 . 0 3 4}$ \\
Greece & - & - & - & - & 0.036 & $\mathbf{0}$ & 0.031 & $\mathbf{0 . 0 6 9}$ & 0.028 & $\mathbf{0 . 0 5 7}$ \\
Spain & - & - & - & - & - & - & 0.120 & $\mathbf{0 . 1 0 3}$ & 0.105 & $\mathbf{0 . 0 9 2}$ \\
Portugal & - & - & - & - & - & - & 0.031 & $\mathbf{0 . 0 6 9}$ & 0.027 & $\mathbf{0 . 0 5 7}$ \\
Austria & - & - & - & - & - & - & - & - & 0.022 & $\mathbf{0 . 0 4 6}$ \\
Sweden & - & - & - & - & - & - & - & - & 0.024 & $\mathbf{0 . 0 4 6}$ \\
Finland & - & - & - & - & - & - & - & - & 0.014 & $\mathbf{0 . 0 3 4}$ \\
\hline
\end{tabular}


Table 11: Population and the nucleolus in the Council of Ministers under QM rules with 15 and 27 members.

\begin{tabular}{lllll}
\hline \hline Country & \multicolumn{2}{c}{ QM 15 } & \multicolumn{2}{c}{ QM 27 } \\
& $\frac{n_{j}}{n}$ & NU & $\frac{n_{j}}{n}$ & NU \\
\hline Germany & 0.219 & $\mathbf{0 . 1 2 2}$ & 0.170 & $\mathbf{0 . 0 8 4}$ \\
France & 0.157 & $\mathbf{0 . 1 2 2}$ & 0.123 & $\mathbf{0 . 0 8 4}$ \\
UK & 0.158 & $\mathbf{0 . 1 2 2}$ & 0.123 & $\mathbf{0 . 0 8 4}$ \\
Italy & 0.154 & $\mathbf{0 . 1 2 2}$ & 0.120 & $\mathbf{0 . 0 8 4}$ \\
Spain & 0.105 & $\mathbf{0 . 1 1 2}$ & 0.082 & $\mathbf{0 . 0 7 8}$ \\
Poland & - & - & 0.080 & $\mathbf{0 . 0 7 8}$ \\
Romania & - & - & 0.047 & $\mathbf{0 . 0 4 1}$ \\
Netherlands & 0.042 & $\mathbf{0 . 0 5 1}$ & 0.033 & $\mathbf{0 . 0 3 8}$ \\
Greece & 0.028 & $\mathbf{0 . 0 5 1}$ & 0.022 & $\mathbf{0 . 0 3 5}$ \\
Portugal & 0.027 & $\mathbf{0 . 0 5 1}$ & 0.021 & $\mathbf{0 . 0 3 5}$ \\
Belgium & 0.027 & $\mathbf{0 . 0 5 1}$ & 0.021 & $\mathbf{0 . 0 3 5}$ \\
Czech Republic & - & - & 0.021 & $\mathbf{0 . 0 3 5}$ \\
Hungary & - & - & 0.021 & $\mathbf{0 . 0 3 5}$ \\
Sweden & 0.024 & $\mathbf{0 . 0 4 1}$ & 0.018 & $\mathbf{0 . 0 2 9}$ \\
Austria & 0.022 & $\mathbf{0 . 0 4 1}$ & 0.017 & $\mathbf{0 . 0 2 9}$ \\
Bulgaria & - & - & 0.017 & $\mathbf{0 . 0 2 9}$ \\
Denmark & 0.014 & $\mathbf{0 . 0 3 1}$ & 0.011 & $\mathbf{0 . 0 2}$ \\
Slovak Republic & - & - & 0.011 & $\mathbf{0 . 0 2}$ \\
Finland & 0.014 & $\mathbf{0 . 0 3 1}$ & 0.011 & $\mathbf{0 . 0 2}$ \\
Ireland & 0.010 & $\mathbf{0 . 0 3 1}$ & 0.008 & $\mathbf{0 . 0 2}$ \\
Lithuania & - & - & 0.008 & $\mathbf{0 . 0 2}$ \\
Latvia & - & - & 0.005 & $\mathbf{0 . 0 1 2}$ \\
Slovenia & - & - & 0.004 & $\mathbf{0 . 0 1 2}$ \\
Estonia & - & - & 0.003 & $\mathbf{0 . 0 1 2}$ \\
Cyprus & - & - & 0.002 & $\mathbf{0 . 0 1 2}$ \\
Luxembourg & 0.001 & $\mathbf{0 . 0 2 0}$ & 0.001 & $\mathbf{0 . 0 1 2}$ \\
Malta & - & - & 0.001 & $\mathbf{0 . 0 0 9}$ \\
\hline
\end{tabular}

It is interesting to compare table 10 with table 5.3.9 in Felsenthal and Machover (1998), where fairness is evaluated using the Banzhaf index as a power measure. By comparing Banzhaf indices and the square root of the population, they show that larger member states tend to have too little power and the smaller ones too much, though they claim that the discrepancies are not too large except for Germany and Luxembourg. In our table we see two types of situation: for 1958, 1986 and 1995 the pattern of larger countries getting a less than proportional payoff is repeated; however in 1973 and 1981 the payoff is divided among 
the four largest countries.

Analogously we can compare table 11 with tables 6 and 9 in Felsenthal and Machover (2001). While larger countries have too little power and smaller countries too much according to the nucleolus, the results are not so clear-cut according to the Banzhaf index. For example, Spain has too much power according to the Banzhaf index but smaller countries like Denmark have too little power.

Obviously, the results suggest that the European citizens are not treated equally under the decision rules operating in the CM since 1958 till now. The reason is that the nucleolus does not coincide with the population ratios. In what follows we investigate the question of whether it would have been possible to do better and describe the methodology to choose the optimal decision rule.

\section{The Optimal (Fair) Decision Rules}

Remark 1 states that if we would like to equalize the citizens' power, we need to choose a voting rule which leads to the nucleolus $\nu_{j}$ for the representatives being equal to the countries' population sizes. However, except in exceptional circumstances, it is not possible to find a game for which the vector of countries' population sizes coincides with the nucleolus. Our tables 10 and 11 provide information on the distance between the first best and the outcome of the choices which were made. We will now show that it was possible to find simple games whose nucleolus is closer to the population shares.

Hereafter, we will assume that the objective of the political architect is to design the simple game $(M, \mathcal{W})$ in such a way that the distance between the induced nucleolus calculated at the citizen level and the egalitarian division is the smallest possible. We chose to measure this distance by the variance, but the minimization of any other inequality index like the Gini index or a Kolm-Atkinson index would also be appropriate.

Denoting by $\mathcal{S}_{m}$ the set of all simple games with $m$ players, our combinatorial problem is defined as follows:

$$
\underset{(M, \mathcal{W}) \in \mathcal{S}_{m}}{\operatorname{Min}} \operatorname{Var}(N U((M, \mathcal{W})))
$$

where

$$
\operatorname{Var}(N U((M, \mathcal{W})))=\sum_{i \in M} n_{i}\left[\frac{1}{n}-\frac{\nu_{i}}{n_{i}}\right]^{2},
$$

where $N U((M, \mathcal{W}))=\left(\nu_{1}, \nu_{2}, \ldots, \nu_{m}\right)$. The term $\frac{\nu_{i}}{n_{i}}$ indicates how much power a citizen in country $i$ gets given a specific voting rule.

Maaser and Napel (2007) refer to (2) as the cumulative individual quadratic deviation and point out that it is equivalent to considering the Euclidean distance. Beisbart and Bovens (2007) also use the quadratic criterion to measure departure from perfect equality.

Expanding (2) we obtain $\operatorname{Var}(N U((M, \mathcal{W})))=\sum_{i \in M} n_{i}\left[\frac{1}{n^{2}}+\frac{\left(\nu_{i}\right)^{2}}{\left(n_{i}\right)^{2}}-\frac{2 \nu_{i}}{n n_{i}}\right]$, or equivalently 
$\sum_{i \in M}\left[\frac{n_{i}}{n^{2}}+\frac{\left(\nu_{i}\right)^{2}}{n_{i}}-\frac{2 \nu_{i}}{n}\right]$. Taking into account that $\sum_{i \in M} n_{i}=n$ and $\sum_{i \in M} \nu_{i}=1$ and collecting terms, we find

$$
\operatorname{Var}(N U((M, \mathcal{W})))=\sum_{i \in M} \frac{\left(\nu_{i}\right)^{2}}{n_{i}}-\frac{1}{n} .
$$

The resolution of our problem would be greatly simplified if we knew the image $\operatorname{Im}\left(N U_{m}\right)$ of the mapping $N U_{m}$ attaching to any simple game $(M, \mathcal{W}) \in \mathcal{S}_{m}$ the nucleolus of the game. $\operatorname{Im}\left(N U_{m}\right)$ is a finite subset of the $(m-1)-$ dimensional simplex. If $\operatorname{Im}\left(N U_{m}\right)$ was characterized, our problem would be

$$
\begin{aligned}
& \operatorname{Min} \sum_{i \in M} \frac{\left(x_{i}\right)^{2}}{n_{i}} \\
& \text { s.t. } x \in \operatorname{Im}\left(N U_{m}\right)
\end{aligned} .
$$

Unfortunately, $\operatorname{Im}\left(N U_{m}\right)$ has not been characterized. The characterization of the set of vectors that can be obtained as power measures for some simple game given the number of players is known as the inverse problem. This problem has been formulated recently by Alon and Edelman (2010) for the Banzhaf index and they obtained partial results. We are not aware of any general result on the inverse problem for the nucleolus. This means that we will examine the combinatorial problem in its original formulation. Due to the large number of simple games, we will restrict the search to a subset $\mathcal{G}_{m}$ of the set $\mathcal{S}_{m}$ of all simple games. The procedure for solving (4) can be presented as the sequence of the following steps:

Step 1. For the given number of countries $m$, list all games in the class $\mathcal{G}_{m}$;

Step 2. Calculate the nucleolus $\nu$ for each game from the list;

Step 3. Find the variance from (3);

Step 4. Choose the game with the minimal variance.

We illustrate our technique for $m=3$. Without loss of generality we assume that $n_{1} \geq n_{2} \geq n_{3}$.

For 3 countries there are very few possible games, so we can solve the problem quite generally. If we assume that the game is monotonic (adding players cannot turn a winning coalition into a losing one), proper (no two disjoint coalitions can be winning), directed (players can be unambiguously ranked in order of desirability with player 1 being at least as desirable as player 2, who is at least as desirable as player 3), $v(\varnothing)=0$ and $v(N)=1$, it turns out that there are only five possible games and three possible values for the nucleolus. All five games can be described as weighted majority games. These are $[1 ; 1,0,0]$ in which player 1 is a dictator, $[3 ; 2,1,1]$ in which player 1 is a veto player but not a dictator, $[2 ; 1,1,0]$ in which players 1 and 2 are veto players, $[3 ; 1,1,1]$ in which all three players are veto players, and $[2 ; 1,1,1]$ which is the simple majority game with no veto players. The nucleolus is $(1,0,0)$ for the first two games, $\left(\frac{1}{2}, \frac{1}{2}, 0\right)$ for the third game and $\left(\frac{1}{3}, \frac{1}{3}, \frac{1}{3}\right)$ for the last two games.

Given (3), the variance for the majority game (and for the unanimity game) is 


$$
\operatorname{Var}_{m a j}=\frac{1}{9}\left[\frac{1}{n_{1}}+\frac{1}{n_{2}}+\frac{1}{n_{3}}\right]-\frac{1}{n},
$$

and the variance for the dictatorial game (and for the game with one veto player) is

$$
\operatorname{Var}_{\text {dict }}=\frac{1}{n_{1}}-\frac{1}{n}
$$

The variance for the rule with two veto players is

$$
\operatorname{Var}_{\text {veto } 12}=\frac{1}{4}\left[\frac{1}{n_{1}}+\frac{1}{n_{2}}\right]-\frac{1}{n}
$$

The next figure shows the values of the two biggest countries' population shares, $\gamma_{1}$ and $\gamma_{2}$, for which each of the three games is optimal.
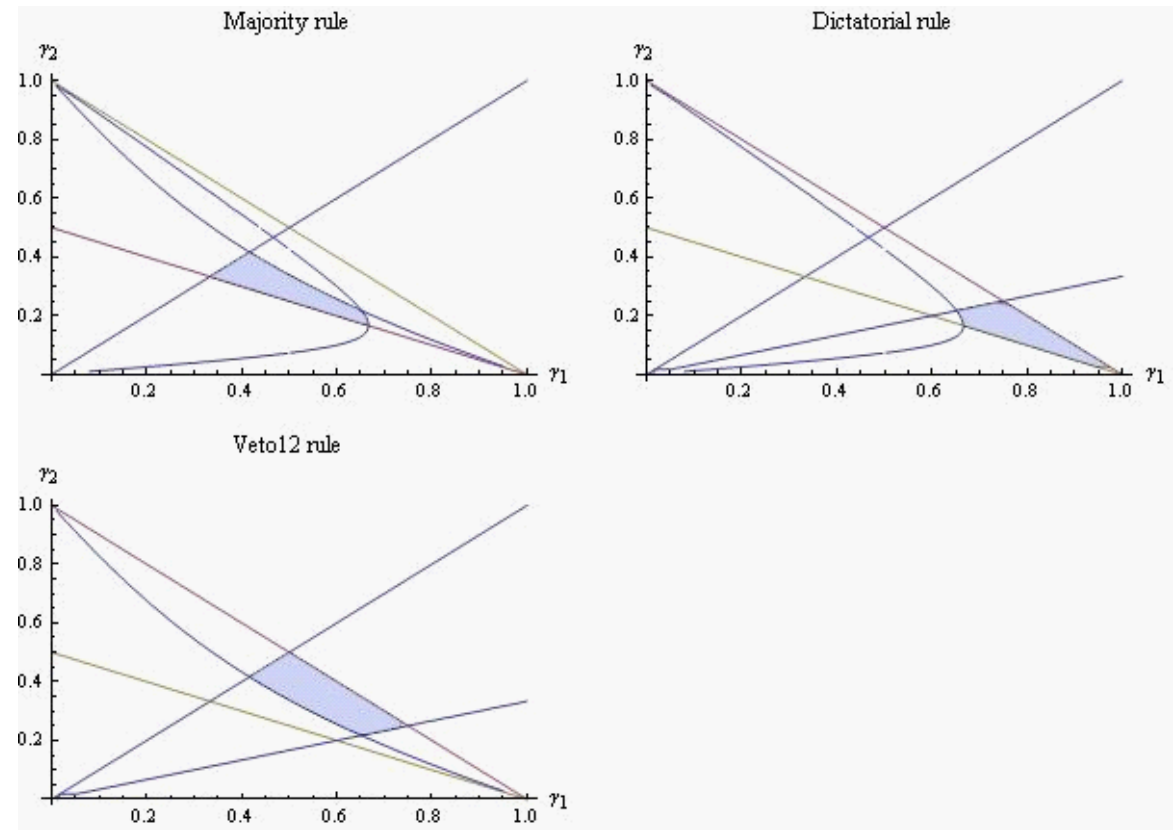

Figure 1: The optimal rule for $m=3$

Not surprisingly, the majority rule is optimal when the three countries are not too different in terms of the population ratios, and the dictatorial rule is optimal in the case where there is a relatively big country.

From the results reported in appendix 3, it is clear that we can solve our optimality problem by "brute force" as long as $m \leq 8$ for some specific important classes of simple games, like constant-sum weighted majority games. It is far from certain that the optimal game will be a member of this class, however this class has some desirable properties. Because the nucleolus is always a representation for constant-sum majority games (Peleg, 1968), by 
choosing voting weights to coincide with the nucleolus we can achieve a maximally transparent voting system in which voting weights are equal to voting power. ${ }^{21}$ Because even this class becomes too large to be analyzed for $m \geq 9$, we also analyze another class of games in which the weights are fixed, so that the only element that varies from game to game is the quota. We then look for the quota that minimizes $n V a r$. We fix the weights to coincide with population shares. This seems a natural choice and it is also the choice made in the Treaty of Lisbon. We implement the following algorithm.

1. Given the vector of weights $\left(\omega_{1}, \ldots, \omega_{n}\right)$, we calculate the total weight $\omega(S)$ associated to each subset $S \subseteq N$. There are $2^{n}$ such subsets (including the empty set).

2. Order the $\omega(S)$ from lowest to largest. About half of these values are above $\frac{\omega(N)}{2}$ (exactly half if none of the coalitions has $\frac{\omega(N)}{2}$ votes). This gives at most $2^{n-1}$ relevant values for the quota. ${ }^{22}$ Any numbers in between two of the values would be equivalent to the higher of the two values and need not be considered.

3. Find the nucleolus for each of the games, calculate $n V a r$, and find the quotas that minimize $n V a r$.

Note that payoffs achievable in this class are not necessarily available in the class of constant-sum weighted majority games and vice versa. For example, in a game with 4 players and $n_{1} \geq n_{2}>n_{3} \geq n_{4}$, setting $q=n_{1}+n_{2}$ leads to a nucleolus payoff of $\left(\frac{1}{2}, \frac{1}{2}, 0,0\right)$, which is not available in the class of constant-sum weighted majority games. On the other hand, with populations $(3,2,2,1)$ the payoff vector $\left(\frac{2}{5}, \frac{1}{5}, \frac{1}{5}, \frac{1}{5}\right)$ is available in the class of constant-sum weighted majority games but cannot be achieved if the weights must coincide with the population shares.

We have chosen these two classes of games for the reasons outlined above. However, there is no guarantee that the optimal game will be a member of either of the two classes. For example, suppose the population values are $(5,4,3,3,2,1)$. If we are restricted to use these values as weights, the best value for the quota is 12 , which leads to a nucleolus value of $\frac{1}{24}(7,6,4,4,2,1)$. If we are restricted to the class of constant-sum weighted majority games, the best choice is $[9 ; 5,4,3,2,2,1]$; weights coincide with population values for all countries except country 4, and the nucleolus is proportional to these weights. We can do quite well within both classes, but we cannot achieve the first best. However, if we take the game $[16 ; 6,5,4,4,3,2]$, which has different weights and is not constant-sum, it turns out that the nucleolus is $\frac{1}{18}(5,4,3,3,2,1)$, exactly proportional to the population values.

In what follows, the nucleolus is computed using Derks and Kuipers' DOS program. The program is available at Jean Derks' homepage (http://www.personeel.unimaas.nl/JeanDerks/), and the algorithm is explained in Derks and Kuipers (1997); see also Wolsey (1976).

\footnotetext{
${ }^{21}$ Życzkowski and Słomczyński (2004) include transparency as a desirable condition for a voting system; see also Pajala (2005).

${ }^{22}$ Because several coalitions may have the same total weight, the number of distinct available quotas may be much lower.
} 


\subsection{The EU Council of Ministers 1958 Revisited}

We now apply our technique to find the optimal decision rule for the EU Council of Ministers in 1958 given the population sizes in Table 10. Table 12 lists all constant-sum weighted majority games with six players (taken from Isbell (1959)) with the corresponding values for the nucleolus and $n V a r$. Note that, as discussed earlier, the nucleolus is proportional to the weights.

Table 12: Constant-Sum Weighted Voting Games with 6 Players.

\begin{tabular}{lll}
\hline \hline & NU & nVar \\
\hline$[1,0,0,0,0,0]$ & $(1,0,0,0,0,0)$ & $\mathbf{2 . 1 0 6}$ \\
{$[1,1,1,0,0,0]$} & $(1 / 3,1 / 3,1 / 3,0,0,0)$ & $\mathbf{0 . 1 4 5}$ \\
{$[2,1,1,1,0,0]$} & $(2 / 5,1 / 5,1 / 5,1 / 5,0,0)$ & $\mathbf{0 . 3 9 1}$ \\
{$[1,1,1,1,1,0]$} & $(1 / 5,1 / 5,1 / 5,1 / 5,1 / 5,0)$ & $\mathbf{0 . 7 7 3}$ \\
{$[3,1,1,1,1,0]$} & $(3 / 7,1 / 7,1 / 7,1 / 7,1 / 7,0)$ & $\mathbf{0 . 4 1 2}$ \\
{$[2,2,1,1,1,0]$} & $(2 / 7,2 / 7,1 / 7,1 / 7,1 / 7,0)$ & $\mathbf{0 . 3 0 5}$ \\
{$[3,2,2,1,1,0]$} & $(1 / 3,2 / 9,2 / 9,1 / 9,1 / 9,0)$ & $\mathbf{0 . 1 2 0}$ \\
{$[2,1,1,1,1,1]$} & $(2 / 7,1 / 7,1 / 7,1 / 7,1 / 7,1 / 7)$ & $\mathbf{1 0 . 2 9 9}$ \\
{$[4,1,1,1,1,1]$} & $(4 / 9,1 / 9,1 / 9,1 / 9,1 / 9,1 / 9)$ & $\mathbf{6 . 2 9 5}$ \\
{$[3,2,1,1,1,1]$} & $(1 / 3,2 / 9,1 / 9,1 / 9,1 / 9,1 / 9)$ & $\mathbf{6 . 1 5 4}$ \\
{$[4,2,2,1,1,1]$} & $(4 / 11,2 / 11,2 / 11,1 / 11,1 / 11,1 / 11)$ & $\mathbf{4 . 0 6 2}$ \\
{$[3,3,2,1,1,1]$} & $(3 / 11,3 / 11,2 / 11,1 / 11,1 / 11,1 / 11)$ & $\mathbf{4 . 0 2 4}$ \\
{$[4,3,3,1,1,1]$} & $(4 / 13,3 / 13,3 / 13,1 / 13,1 / 13,1 / 13)$ & $\mathbf{2 . 8 3 7}$ \\
{$[5,2,2,2,1,1]$} & $(5 / 13,2 / 13,2 / 13,2 / 13,1 / 13,1 / 13)$ & $\mathbf{3 . 0 5 9}$ \\
{$[5,3,3,2,1,1]$} & $(1 / 3,1 / 5,1 / 5,2 / 15,1 / 15,1 / 15)$ & $\mathbf{2 . 2 0 8}$ \\
{$[2,2,2,1,1,1]$} & $(2 / 9,2 / 9,2 / 9,1 / 9,1 / 9,1 / 9)$ & $\mathbf{6 . 1 0 2}$ \\
{$[3,2,2,2,1,1]$} & $(3 / 11,2 / 11,2 / 11,2 / 11,1 / 11,1 / 11)$ & $\mathbf{4 . 2 5 8}$ \\
{$[4,3,2,2,1,1]$} & $(4 / 13,3 / 13,2 / 13,2 / 13,1 / 13,1 / 13)$ & $\mathbf{2 . 9 9 5}$ \\
{$[3,3,2,2,2,1]$} & $(3 / 13,3 / 13,2 / 13,2 / 13,2 / 13,1 / 13)$ & $\mathbf{3 . 2 0 1}$ \\
{$[4,3,3,2,2,1]$} & $(4 / 15,1 / 5,1 / 5,2 / 15,2 / 15,1 / 15)$ & $\mathbf{2 . 3 3 6}$ \\
{$[5,4,3,2,2,1]$} & $(5 / 17,4 / 17,3 / 17,2 / 17,2 / 17,1 / 17)$ & $\mathbf{1 . 7 7 7}$ \\
\hline \hline
\end{tabular}

As one can see from Table 12, in the class of constant-sum weighted majority games the game $[3,2,2,1,1,0]$ provides the minimal variance with $n V a r=0.12$. The actual decision rule for 1958 is not in the list, because it is not a strong game. However, $n V$ ar for this game equals 0.175 , and therefore this rule cannot be optimal.

Two conclusions can be drawn from this exercise. First, Germany got too little weight compared to France and Italy. Second, the choice to make Luxembourg a dummy was optimal in our context: because the population of Luxembourg is very small compared with 
the rest of countries, all games in which Luxembourg has a positive payoff have a very large variance.

If instead we make weights coincide with population shares, the vector of weights would be $(0.322,0.291,0.266,0.066,0.053,0.002)$. There are $2^{5}=32$ possible games, but only 9 different values for the nucleolus. Perhaps surprisingly, the optimal voting game in this class has the same nucleolus and thus the same $n V$ ar as the optimal voting game in the class of constant-sum weighted majority games.

Table 13: Possible Values for the Nucleolus in the 1958 Council of Ministers using the Population as Weights.

\begin{tabular}{lll}
\hline \hline Quota & NU & nVar \\
\hline$(0.500,0.625]$ & $(1 / 3,1 / 3,1 / 3,0,0,0)$ & $\mathbf{0 . 1 4 5}$ \\
$(0.625,0.643]$ & $(1 / 3,2 / 9,2 / 9,1 / 9,1 / 9,0)$ & $\mathbf{0 . 1 2 0}$ \\
$(0.643,0.656]$ & $(1 / 3,1 / 4,1 / 6,1 / 6,1 / 12,0)$ & $\mathbf{0 . 2 1 6}$ \\
$(0.656,0.668]$ & $(2 / 7,2 / 7,1 / 7,1 / 7,1 / 7,0)$ & $\mathbf{0 . 3 0 5}$ \\
$(0.668,0.678]$ & $(1 / 4,1 / 4,1 / 4,1 / 4,0,0)$ & $\mathbf{0 . 5 9 1}$ \\
$(0.678,0.709]$ & $(1,0,0,0,0,0)$ & $\mathbf{2 . 1 0 6}$ \\
$(0.709,0.734]$ & $(0.5,0.5,0,0,0,0)$ & $\mathbf{0 . 6 3 6}$ \\
$(0.734,0.934]$ & $(1 / 3,1 / 3,1 / 3,0,0,0)$ & $\mathbf{0 . 1 4 5}$ \\
$(0.934,0.947]$ & $(1 / 4,1 / 4,1 / 4,1 / 4,0,0)$ & $\mathbf{0 . 5 9 1}$ \\
$(0.947,0.998]$ & $(1 / 5,1 / 5,1 / 5,1 / 5,1 / 5,0)$ & $\mathbf{0 . 7 7 3}$ \\
$(0.998,1]$ & $(1 / 6,1 / 6,1 / 6,1 / 6,1 / 6,1 / 6)$ & $\mathbf{1 4 . 1 2 0}$ \\
\hline \hline
\end{tabular}

\subsection{The EU Council of Ministers 1973 Revisited}

The 1973 Council of Ministers has nine states. As can be seen in table 17, there are 175428 constant-sum weighted majority games with $n=9$.

If we assume that weights coincide with population shares, we find 201 possible games and 33 different values for the nucleolus. The optimal value of the quota is in the interval $(0.554,0.563]$, leading to payoff vector $\left(\frac{4}{15}, \frac{3}{15}, \frac{3}{15}, \frac{3}{15}, \frac{1}{15}, \frac{1}{15}, 0,0,0\right)$ and $n V a r=0.064$. This is again lower than the value for the actual decision rule in 1973, which is $n V a r=0.145$. As in the 1958 case, the nucleolus of the optimal game treats Germany differently from the other large countries, and the other large countries are treated symmetrically ${ }^{23}$. Also, some of the states receive 0 in the nucleolus of the optimal game ${ }^{24}$.

\footnotetext{
${ }^{23}$ If we order the possible values of the nucleolus by decreasing nVar, the first few values also have the property that Germany gets more than the next largest country.

${ }^{24}$ Unlike in the 1958 case, there are no dummy players in the optimal game. Note that there are values of the quota for which each of the nine countries gets a different payoff. It is almost inevitable for Luxembourg to get 0 (the only exception is when unanimity is required), but there are quota values for which the other small countries would get a positive payoff.
} 
Table 14: Possible Values for the Nucleolus in the 1973 Council of Ministers using the Population as Weights.

\begin{tabular}{|c|c|c|}
\hline Quota & $\mathrm{NU}$ & nVar \\
\hline$[0.500,0.503]$ & $\left., \frac{4}{39}, \frac{3}{39}, \frac{2}{39}, \frac{1}{39}, 0\right)$ & 0.186 \\
\hline$(0.503,0.505]$ & $\left.\frac{2}{27}, \frac{1}{27}, \frac{1}{27}, 0\right)$ & 0.198 \\
\hline$(0.505,0.508]$ & $\left.\frac{3}{38}, \frac{1}{38}, \frac{1}{38}, 0\right)$ & 0.140 \\
\hline$(0.508,0.510]$ & $\left.\frac{7}{95}, \frac{3}{95}, \frac{2}{95}, 0\right)$ & 0.125 \\
\hline$(0.510,0.511]$ & $\left.\frac{5}{11}, \frac{4}{51}, \frac{2}{51}, \frac{1}{51}, 0\right)$ & 0.132 \\
\hline$(0.511,0.512]$ & $\left.\frac{5}{68}, \frac{3}{68}, \frac{1}{68}, 0\right)$ & 0.137 \\
\hline$(0.512,0.513]$ & $\left.\frac{3}{32}, \frac{2}{32}, \frac{1}{32}, \frac{1}{32}, 0\right)$ & 0.109 \\
\hline$(0.513,0.515]$ & $\left.\frac{4}{43}, \frac{3}{43}, \frac{2}{43}, \frac{1}{43}, 0\right)$ & 0.128 \\
\hline$(0.515,0.516]$ & $\left.\frac{4}{26}, \frac{3}{26}, \frac{2}{26}, \frac{1}{26}, 0,0\right)$ & 0.169 \\
\hline$(0.516,0.518]$ & $\left.\frac{2}{11}, \frac{1}{11}, \frac{1}{11}, 0,0,0\right)$ & 0.153 \\
\hline$(0.518,0.524]$ & $\left.\frac{1}{31}, \frac{1}{31}, 0\right)$ & 0.117 \\
\hline$(0.524,0.525]$ & $\left.\frac{1}{20}, 0\right)$ & 0.129 \\
\hline$(0.525,0.526]$ & $\left.\frac{3}{47}, \frac{2}{47}, \frac{1}{47}, 0\right)$ & 0.088 \\
\hline$(0.526,0.527]$ & $0,0)$ & 0.178 \\
\hline$(0.527,0.528]$ & $\left.\frac{1}{72}, \frac{1}{72}, 0\right)$ & 0.089 \\
\hline$(0.528,0.529]$ & $\left.\frac{2}{34}, \frac{1}{34}, \frac{1}{34}, 0\right)$ & 0.081 \\
\hline$(0.529,0.530]$ & $\left.\frac{1}{25}, 0,0\right)$ & 0.110 \\
\hline$(0.530,0.544]$ & $, 0,0)$ & 0.502 \\
\hline$(0.544,0.554]$ & $\left.\frac{1}{0}, \frac{1}{10}, 0,0,0\right)$ & 0.188 \\
\hline$(0.554,0.563]$ & $\left.\frac{1}{15}, 0,0,0\right)$ & 0.064 \\
\hline$(0.563,0.567]$ & $\left.\frac{1}{20}, \frac{1}{20}, \frac{1}{20}, 0,0\right)$ & 0.071 \\
\hline$(0.567,0.578]$ & $, 0,0,0,0)$ & 0.488 \\
\hline, 0.582$]$ & $0,0,0,0)$ & 0.235 \\
\hline$(0.582,0.733]$ & $, 0,0,0,0)$ & 0.145 \\
\hline$(0.733,0.738]$ & $0,0,0,0)$ & 0.214 \\
\hline$(0.738,0.739]$ & $\left.\frac{3}{24}, \frac{1}{24}, \frac{1}{24}, 0,0\right)$ & 0.175 \\
\hline$(0.739,0.743]$ & $\left.\frac{1}{20}, \frac{1}{20}, \frac{1}{20}, 0,0\right)$ & 0.129 \\
\hline$(0.743,0.744]$ & $\left.\frac{2}{18}, \frac{1}{28}, 0,0\right)$ & 0.141 \\
\hline$(0.744,0.757]$ & $, 0,0,0,0)$ & 0.502 \\
\hline$(0.757,0.781]$ & $, 0,0,0,0,0,0,0)$ & 3.132 \\
\hline$(0.781,0.785]$ & $0,0,0,0,0,0,0)$ & 1.180 \\
\hline$(0.785,0.796]$ & $0,0,0,0,0,0)$ & 0.488 \\
\hline$(0.796,0.947]$ & $0,0,0,0,0)$ & 0.145 \\
\hline$(0.947,0.961]$ & $\left.\frac{1}{5}, 0,0,0,0\right)$ & 0.502 \\
\hline$(0.961,0.980]$ & $\left.\frac{1}{6}, \frac{1}{6}, 0,0,0\right)$ & 0.774 \\
\hline$(0.980,0.987]$ & $\left., \frac{1}{7}, 0,0\right)$ & 1.377 \\
\hline$(0.987,0.988]$ & $\left.\frac{1}{8}, 0\right)$ & 2.122 \\
\hline$(0.988,0.999]$ & $\left.\frac{1}{9}\right)$ & 13.813 \\
\hline
\end{tabular}


Table 15: Possible Values for the Nucleolus in the 1981 Council of Ministers using the Population as Weights.

\begin{tabular}{|c|c|c|}
\hline Quota & 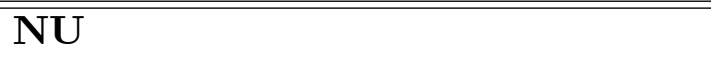 & "nVar \\
\hline$[0.500,0.501]$ & $\left.\frac{3}{7}, \frac{19}{107}, \frac{18}{107}, \frac{17}{107}, \frac{10}{107}, \frac{7}{107}, \frac{7}{107}, \frac{4}{107}, \frac{2}{107}, 0\right)$ & 0.121 \\
\hline$(0.501,0.502]$ & $\left(\frac{7}{33}, \frac{6}{33}, \frac{6}{33}, \frac{5}{33}, \frac{3}{33}, \frac{2}{33}, \frac{2}{33}, \frac{1}{33}, \frac{1}{33}, 0\right)$ & 0.111 \\
\hline$(0.502,0.507]$ & $\left.\frac{5}{9}, \frac{5}{29}, \frac{5}{29}, \frac{3}{29}, \frac{2}{29}, \frac{2}{29}, \frac{1}{29}, 0,0\right)$ & 0.152 \\
\hline$(0.507,0.509]$ & $\left.\frac{8}{48}, \frac{4}{48}, \frac{3}{48}, \frac{3}{48}, \frac{2}{48}, \frac{1}{48}, 0\right)$ & 0.106 \\
\hline$(0.509,0.511]$ & $\left(\frac{7}{34}, \frac{6}{34}, \frac{6}{34}, \frac{6}{34}, \frac{3}{34}, \frac{2}{34}, \frac{2}{34}, \frac{1}{34}, \frac{1}{34}, 0\right)$ & 0.094 \\
\hline$(0.511,0.516]$ & $\left.\frac{5}{32}, \frac{3}{32}, \frac{2}{32}, \frac{2}{32}, \frac{1}{32}, 0,0\right)$ & 0.106 \\
\hline$(0.516,0.519]$ & $\left.\frac{10}{54}, \frac{9}{54}, \frac{4}{54}, \frac{3}{54}, \frac{3}{54}, \frac{2}{54}, \frac{1}{54}, 0\right)$ & 0.060 \\
\hline$(0.519,0.522]$ & $\left.\frac{6}{77}, \frac{3}{37}, \frac{2}{37}, \frac{2}{37}, \frac{1}{37}, \frac{1}{37}, 0\right)$ & 0.063 \\
\hline$(0.522,0.524]$ & $\left.\frac{6}{33}, \frac{3}{33}, \frac{2}{33}, \frac{2}{33}, \frac{1}{33}, 0,0\right)$ & 0.090 \\
\hline$(0.524,0.528]$ & $\left.\frac{9}{51}, \frac{4}{51}, \frac{3}{51}, \frac{3}{51}, \frac{1}{51}, \frac{1}{51}, 0\right)$ & 0.054 \\
\hline$(0.528,0.530]$ & $\left.\frac{7}{38}, \frac{3}{38}, \frac{2}{38}, \frac{2}{38}, \frac{1}{38}, \frac{1}{38}, 0\right)$ & 0.053 \\
\hline$(0.530,0.534]$ & $\left.\frac{7}{36}, \frac{6}{36}, \frac{3}{36}, \frac{2}{36}, \frac{2}{36}, \frac{1}{36}, 0,0\right)$ & 0.064 \\
\hline$(0.534,0.537]$ & $\left.\frac{3}{16}, \frac{1}{16}, \frac{1}{16}, \frac{1}{16}, 0,0,0\right)$ & 0.080 \\
\hline$(0.537,0.541]$ & $\left.\frac{6}{32}, \frac{6}{32}, \frac{2}{32}, \frac{2}{32}, \frac{2}{32}, \frac{1}{32}, 0,0\right)$ & 0.067 \\
\hline$(0.541,0.543]$ & $5,0,0,0,0,0)$ & 0.517 \\
\hline$(0.543,0.562]$ & $\left.\frac{3}{15}, \frac{1}{15}, \frac{1}{15}, \frac{1}{15}, 0,0,0\right)$ & 0.093 \\
\hline$(0.562,0.573]$ & $\left.\frac{4}{20}, \frac{1}{20}, \frac{1}{20}, \frac{1}{20}, 0,0,0\right)$ & 0.047 \\
\hline$(0.573,0.577]$ & $\left.\frac{5}{25}, \frac{5}{25}, \frac{1}{25}, \frac{1}{25}, \frac{1}{25}, \frac{1}{25}, 0,0\right)$ & 0.042 \\
\hline$(0.577,0.585]$ & $\left.\frac{6}{30}, \frac{6}{30}, \frac{6}{30}, \frac{1}{30}, \frac{1}{30}, \frac{1}{30}, \frac{1}{30}, \frac{1}{30}, 0\right)$ & 0.052 \\
\hline$(0.585,0.590]$ & $\left.\frac{1}{3}, 0,0,0,0,0,0,0\right)$ & 0.561 \\
\hline$(0.590,0.594]$ & $0,0,0,0,0,0)$ & 0.293 \\
\hline$(0.594,0.614]$ & $, 0,0,0,0,0)$ & 0.191 \\
\hline$(0.614,0.737]$ & $\left.\frac{3}{16}, \frac{1}{16}, \frac{1}{16}, \frac{1}{16}, 0,0,0\right)$ & 0.080 \\
\hline$(0.737,0.744]$ & $\left.\frac{12}{76}, \frac{7}{76}, \frac{4}{76}, \frac{4}{76}, \frac{2}{76}, \frac{1}{76}, 0\right)$ & 0.060 \\
\hline$(0.744,0.746]$ & $\left(\frac{16}{72}, \frac{14}{72}, \frac{13}{72}, \frac{12}{72}, \frac{6}{72}, \frac{4}{72}, \frac{4}{72}, \frac{2}{72}, \frac{1}{72}, 0\right)$ & 0.053 \\
\hline$(0.746,0.747]$ & $\left.\frac{6}{37}, \frac{3}{37}, \frac{2}{37}, \frac{2}{37}, \frac{1}{37}, \frac{1}{37}, 0\right)$ & 0.063 \\
\hline$(0.747,0.750]$ & $\left.\frac{1}{5}, \frac{1}{5}, \frac{1}{5}, 0,0,0,0,0\right)$ & 0.517 \\
\hline$(0.750,0.767]$ & $\left(\frac{3}{15}, \frac{3}{15}, \frac{3}{15}, \frac{3}{15}, \frac{1}{15}, \frac{1}{15}, \frac{1}{15}, 0,0,0\right)$ & 0.093 \\
\hline$(0.767,0.775]$ & $(1,0,0,0,0,0,0,0,0,0)$ & 3.386 \\
\hline$(0.775,0.794]$ & $, 0,0,0,0,0,0,0)$ & 1.293 \\
\hline$(0.794,0.799]$ & $\left.\frac{1}{3}, 0,0,0,0,0,0,0\right)$ & 0.561 \\
\hline$(0.799,0.842]$ & $\left.\frac{1}{4}, 0,0,0,0,0,0\right)$ & 0.191 \\
\hline$(0.842,0.950]$ & $\left., \frac{1}{5}, 0,0,0,0,0\right)$ & 0.517 \\
\hline$(0.950,0.967]$ & $\left., \frac{1}{7}, \frac{1}{7}, 0,0,0\right)$ & 0.908 \\
\hline$(0.967,0.986]$ & $\left.\frac{1}{8}, \frac{1}{8}, 0,0\right)$ & 1.283 \\
\hline$(0.986,0.999]$ & $\left.\frac{1}{9}, \frac{1}{9}, \frac{1}{9}, \frac{1}{9}, \frac{1}{9}, 0\right)$ & 1.753 \\
\hline$(0.999,1]$ & $\left(\frac{1}{10}, \frac{1}{10}, \frac{1}{10}, \frac{1}{10}, \frac{1}{10}, \frac{1}{10}, \frac{1}{10}, \frac{1}{10}, \frac{1}{10}, \frac{1}{10}\right)$ & 11.230 \\
\hline
\end{tabular}




\subsection{The EU Council of Ministers 1981 Revisited}

In principle, if we fix the weights and vary the quota there could be up to $2^{9}=512$ different weighted majority games with ten players. However, given the population shares $w=(0.228,0.209,0.205,0.200,0.053,0.036,0.036,0.019,0.013,0.001)$ there is a substantial duplication of values for $w(S) .{ }^{25}$ It turns out that two countries have the same (rounded) population shares $\left(\omega_{6}=\omega_{7}\right)$. Moreover, there are groups of countries with the same total weight (e.g., $\omega_{1}=\omega_{2}+\omega_{8}, \omega_{1}+\omega_{9}=\omega_{3}+\omega_{6}, \omega_{5}+\omega_{8}=\omega_{6}+\omega_{7}, \omega_{4}+\omega_{8}=\omega_{3}+\omega_{9}+\omega_{10}$ ). Because of this, there are only 239 different games, which correspond to 30 different values of the nucleolus. The optimal value of the quota is in the interval $(0.573,0.577]$, leading to payoff vector $\left(\frac{6}{25}, \frac{5}{25}, \frac{5}{25}, \frac{5}{25}, \frac{1}{25}, \frac{1}{25}, \frac{1}{25}, \frac{1}{25}, 0,0\right)$ and $n V a r=0.042$. This is an improvement over the actual decision rule in 1981, which has $n V a r=0.190$. As before, it turns out that the nucleolus of the optimal game gives Germany a larger payoff, whereas the other three large countries are treated symmetrically. ${ }^{26}$

The optimal values for the nucleolus are summarized in table 16.

Table 16: Optimal values for the nucleolus if weights must coincide with population shares.

\begin{tabular}{lllllll}
\hline \hline Country & \multicolumn{2}{c}{$\mathbf{1 9 5 8}$} & \multicolumn{2}{c}{$\mathbf{1 9 7 3}$} & \multicolumn{2}{c}{$\mathbf{1 9 8 1}$} \\
& $\frac{n_{j}}{n}$ & $\mathbf{N U}$ & $\frac{n_{j}}{n}$ & NU & $\frac{n_{j}}{n}$ & NU \\
\hline France & 0.266 & $\mathbf{0 . 2 2 2}$ & 0.203 & $\mathbf{0 . 2}$ & 0.200 & $\mathbf{0 . 2}$ \\
Germany & 0.322 & $\mathbf{0 . 3 3 3}$ & 0.242 & $\mathbf{0 . 2 6 7}$ & 0.228 & $\mathbf{0 . 2 4}$ \\
Italy & 0.291 & $\mathbf{0 . 2 2 2}$ & 0.214 & $\mathbf{0 . 2}$ & 0.209 & $\mathbf{0 . 2}$ \\
Belgium & 0.053 & $\mathbf{0 . 1 1 1}$ & 0.038 & $\mathbf{0 . 0 6 7}$ & 0.036 & $\mathbf{0 . 0 4}$ \\
Netherlands & 0.066 & $\mathbf{0 . 1 1 1}$ & 0.052 & $\mathbf{0 . 0 6 7}$ & 0.053 & $\mathbf{0 . 0 4}$ \\
Luxembourg & 0.002 & $\mathbf{0}$ & 0.001 & $\mathbf{0}$ & 0.001 & $\mathbf{0}$ \\
UK & - & - & 0.218 & $\mathbf{0 . 2}$ & 0.205 & $\mathbf{0 . 2}$ \\
Denmark & - & - & 0.019 & $\mathbf{0}$ & 0.019 & $\mathbf{0 . 0 4}$ \\
Ireland & - & - & 0.012 & $\mathbf{0}$ & 0.013 & $\mathbf{0}$ \\
Greece & - & - & - & - & 0.036 & $\mathbf{0 . 0 4}$ \\
\hline
\end{tabular}

Using the Gini coefficient instead of $n V$ ar as a measure of inequality would give very similar results ${ }^{27}$. The optimal value for the nucleolus in 1958 and 1981 is not affected. For

\footnotetext{
${ }^{25}$ This duplication is due to the rounding of population shares. We have rounded the population shares to three decimal places and then worked with the rounded weights. If instead we take the population values reported by Felsenthal and Machover (which are also rounded, but less so) and calculate $\omega(S)$ for all coalitions, it turns out that all 1024 values are distinct. On the other hand, with weights rounded to three decimal places there cannot be more than 1000 distinct values, and in fact there are only 479 distinct values.

${ }^{26} \mathrm{It}$ is also the case that if we order the possible values of the nucleolus by decreasing nVar, the first few values also have the property that Germany gets more than the next largest country.

${ }^{27}$ An ideal robustness test would involve the calculation of the the Lorenz curve (Van Puyenbroeck (2008)) attached to each simple game. The pointwise comparison of these curves produces a partial ordering of the simple games which does not depend upon the specific details of the disproportionality index which is used (Monroe (1994)).
} 
1973, the two nucleolus values with the lowest $n V$ ar also have the lowest Gini coefficient, but the order is reversed. The payoff vector that minimizes the Gini index gives a positive payoff of 0.05 to Denmark at the expense of Germany, Belgium and the Netherlands (whose payoffs are reduced to 0.25 for Germany and 0.05 for Belgium and the Netherlands). All other payoffs are unchanged.

One may also ask whether, taking the actual weights as given, the choice of the quota was optimal. It turns out that the quota of 41 could not be improved for the 1973 Council given the actual weights $(10,10,10,10,5,5,3,3,2)$, but there could have been improvements in the other two Councils. For the 1958 Council of Ministers, if weights are fixed at their actual values $(4,4,4,2,2,1)$, the quota of 12 yields a nucleolus of $\left(\frac{1}{4}, \frac{1}{4}, \frac{1}{4}, \frac{1}{8}, \frac{1}{8}, 0\right)$. The payoff vector $\left(\frac{1}{3}, \frac{1}{3}, \frac{1}{3}, 0,0,0\right)$ has a lower value for $n V a r$, and it is the nucleolus of the game for a quota of 10, 14 or 15. Neither was the quota of 45 optimal for the 1981 Council of Ministers given the actual weights $(10,10,10,10,5,5,5,3,3,2)$ : it yields a nucleolus of $\left(\frac{1}{4}, \frac{1}{4}, \frac{1}{4}, \frac{1}{4}, 0,0,0,0,0,0\right)$, but a quota of 46 yields $\left(\frac{5}{28}, \frac{5}{28}, \frac{5}{28}, \frac{5}{28}, \frac{2}{28}, \frac{2}{28}, \frac{2}{28}, \frac{1}{28}, \frac{1}{28}, 0\right)$, which corresponds to a lower value of $n V a r$.

\section{Conclusion}

In this paper, we have developed a methodology to evaluate and design voting organizations in order to minimize the distance to an egalitarian sharing of the surplus when the process of division across the countries which are members of the organization is described by the nucleolus of the simple game. We have explained why the vector corresponding to the nucleolus can be viewed as a vector measuring the power of each member of the organization when the policy issue has the characteristics of distributive politics.

In the first part of the paper, we have reported our computation results concerning the nucleolus for the organizations describing the five consecutive stages of the EU. For the 1958, 1986 and 1995 cases we reach a similar conclusion to studies that use the Banzhaf index: smaller countries tend to have a disproportionately high power. For 1973 and 1981 we reach the opposite conclusion: it is the larger countries that are favored. We have also formulated the design issue and alluded to the difficulties attached to the resulting combinatorial problem. For the sake of illustration, we have shown our optimization at work in the class of constant-sum weighted majority games in the case of the EU in 1958. Among the lessons of this exercise, we were able to confirm that making Luxembourg a dummy was appropriate but that Germany was mistreated. This conclusion is not affected if we consider the class of games in which weights coincide with population shares. Since this latter class is smaller than the class of constant-sum weighted majority games, we were able to perform the optimization exercise for 1973 and 1981 as well. In both cases it turns out that the optimal game assigns Germany the largest payoff, the three other large countries get the same payoff, and some other smaller countries get 0 . The same qualitative results are achieved if the Gini coefficient is used instead of the variance. 


\section{Appendix}

\subsection{Appendix 1 : Cooperative Games $^{28}$, Least Core and Nucleolus}

A cooperative game with transferable utility (TU) is a pair $(N, V)$ where $N=\{1, \ldots, n\}$ with $n \geq 2$ is a finite set of players and $V$ is a function that associates a real number $V(S)$ to each subset $S$ of $N$. It is assumed that $V(\varnothing)=0$. The game is constant-sum if $V(S)+$ $V(N \backslash S)=V(N)$. It is monotonic if $S \subseteq T \subseteq N \Rightarrow V(S) \leq V(T)$. It is zero-monotonic if $V(S \cup\{i\})-V(S) \geq V(\{i\})$ for all $i \in N$ and $S \subseteq N \backslash\{i\}$. It is superadditive if $V(S \cup T) \geq$ $V(S)+V(T)$ for all $S, T \subseteq N$ such that $S \cap T=\varnothing$. A player $i \in N$ is a null-player (dummy) of $(N, V)$ if $V(S \cup\{i\})=V(S)(V(S \cup\{i\})=V(S)+V\{i\})$. Hereafter, we denote by $X_{P O} \equiv$ $\left\{y \in \mathbb{R}^{n} \mid \sum_{i=1}^{n} y^{i}=V(N)\right\}$ the set of preimputations (or Pareto optimal imputations) and by $X_{I R} \equiv\left\{y \in \mathbb{R}^{n} \mid \sum_{i=1}^{n} y^{i}=V(N), y^{j} \geq V(\{j\}) \forall j \in N\right\}$ the set of imputations i.e. the set of individually rational preimputations. A player $k \in N$ is at least as desirable as a player $l \in N$, denoted $k \succeq l$ if $V(S \cup\{k\}) \geq V(S \cup\{l\})$ for all $S \subseteq N \backslash\{k, l\}$. The desirability relation $\succeq$ is reflexive and transitive. If $\succeq$ is complete, the game is called a complete game. According to Krohn and Sudhölter (1995), a directed game is a complete game such that $1 \succeq 2 \succeq \ldots \succeq n$.

Let $X$ be a compact and convex subset of $\mathbb{R}^{n}$ and let $x \in X$. We denote by $\theta(x)$ the $2^{n}$-dimensional vector whose components are the excesses $e(S, x) \equiv V(S)-\sum_{i \in S} x^{i}$ for $\emptyset \subseteq S \subseteq N$ arranged according to their magnitude, i.e., $\theta^{i}(x) \geq \theta^{j}(x)$ for $1 \leq i \leq j \leq 2^{n}$. The nucleolus of $(N, V)$ with respect to $X$ is the unique vector ${ }^{29} x^{*}=N_{u}(N, V) \in X$ such that $\theta\left(x^{*}\right)$ is minimal, in the sense of the lexicographic order, in the set $\{\theta(y) \mid y \in X\}$. The nucleolus of $(N, V)$ with respect to $X_{I R}$ will be called hereafter the nucleolus; it is the nucleolus as originally defined by Schmeidler $(1969)^{30}$. We denote by $\psi(x)$ the $2^{2 n}$ dimensional vector whose components are the numbers $e(S, x)-e(T, x)$ for $\emptyset \subseteq S, T \subseteq N$ arranged according to their magnitude, i.e., $\psi^{i}(x) \geq \psi^{j}(x)$ for $1 \leq i \leq j \leq 2^{2 n}$. The modiclus (Sudhölter (1997)) is the unique vector $x^{* *} \in X_{P O}$ such that $\psi\left(x^{* *}\right)$ is minimal, in the sense of the lexicographic order, in the set $\left\{\psi(y) \mid y \in X_{P O}\right\}$.

Given a real number $\epsilon$, the $\epsilon$-core of $(N, V)$ is the set

$$
C_{\epsilon} \equiv\left\{x \in X_{P O}: e(S, x) \leq \epsilon \text { for all } \varnothing \varsubsetneqq S \varsubsetneqq N\right\} \text {. }
$$

The least core of $(N, V)$, introduced by Maschler, Peleg and Shapley (1979) and denoted $L C(V, N)$, is the intersection of all nonempty $\epsilon$-cores of $(N, V)$. If $(N, V)$ is zero-monotonic, then $L C(V, N) \subseteq X_{I R}$. Then $L C(V, N)$ consists of the vectors $x$ such that $\theta_{1}(x)=\theta_{1}\left(x^{*}\right)$ and consequently $x^{*} \in L C(V, N)$.

\footnotetext{
${ }^{28}$ See Owen (1995) and Peleg and Sudhölter (2003).

${ }^{29}$ See Peleg and Sudhölter (2003) for a proof of uniqueness.

${ }^{30}$ In contrast, the prenucleolus is the nucleolus with respect to $X_{I R}$. If the game is zero-monotonic the nucleolus and the prenucleolus coincide. A simple game is always zero-monotonic unless $\{i\}, S \in \mathcal{W}$ for some $i \in N$ and $S \subseteq N \backslash\{i\}$.
} 


\subsection{Appendix 2 : Simple Games ${ }^{31}$}

A simple game is a pair $(N, \mathcal{W})$ where $N=\{1, \ldots, n\}$ with $n \geq 2$ is a finite set of players and $\mathcal{W}$ is a set of subsets of $N$ satisfying : $N \in \mathcal{W}, \varnothing \notin \mathcal{W}$ and the monotonicity condition $(S \subseteq T \subseteq N$ and $S \in \mathcal{W}) \Rightarrow T \in \mathcal{W}$. The collection $\mathcal{W}$ of coalitions is the set of winning coalitions. The simple game $(N, \mathcal{W})$ is proper if $S \in \mathcal{W} \Rightarrow N \backslash S \notin \mathcal{W}$. It is strong if $S \notin \mathcal{W} \Rightarrow N \backslash S \in \mathcal{W}$. It is constant sum (self-dual or decisive) if it is proper and strong ${ }^{32}$. Hereafter, we will attach to any simple $(N, \mathcal{W})$ the monotonic TU cooperative game $(N, V)$ where

$$
V(S)=\left\{\begin{array}{l}
1 \text { if } S \in W \\
0 \text { otherwise }
\end{array} .\right.
$$

Note that $(N, V)$ is superadditive iff $(N, \mathcal{W})$ is proper and that $(N, V)$ is constant-sum iff $(N, \mathcal{W})$ is decisive. A simple game $(N, \mathcal{W})$ is a weighted majority game if there exists a vector $\omega=\left(q ; \omega_{1}, \ldots, \omega_{n}\right)$ of $(n+1)$ nonnegative real numbers such that a coalition $S \in \mathcal{W} \Longleftrightarrow \sum_{i \in S} \omega_{i} \geq q ; q$ is referred to as the quota and $\omega_{i}$ is the weight of player $i \in N$. The vector $\omega$ is called a representation of the simple game $(N, \mathcal{W})$; the same game may admit several representations. Note that if $\omega_{i} \geq \omega_{j}$, then player $i$ is at least as desirable as a player $j$. A simple game is homogeneous if there exists a representation $\omega$ such that $\sum_{i \in S} \omega_{i}=\sum_{i \in T} \omega_{i}$ for all $S, T \in \mathcal{W}_{m}$ where $\mathcal{W}_{m}$ denotes the set of minimal winning coalitions; such a representation is called a homogeneous representation. A representation is symmetric if $\omega_{i}=\omega_{j}$ whenever $i$ and $j$ are interchangeable.

The dual of $(N, \mathcal{W})$ is the simple game $(N, \mathcal{B})$ where $S \in \mathcal{B}$ if and only if $N \backslash S \notin \mathcal{W}$. The collection $\mathcal{B}$ of coalitions is the set of blocking coalitions.

\subsection{Appendix 3: Representation and Enumeration of Simple Games}

A representation of a weighted majority game $(N, \mathcal{W})$ is an integral representation if $\omega^{i} \in$ $\mathbb{N} \cup\{0\}$ for all $i \in N$. Note that, without loss of generality, the quota $q$ can be chosen to be $\underset{S \in \mathcal{W}_{m}}{\operatorname{Min}} \omega(S)$. An integral representation $\omega$ is minimal if there does not exist another integral representation $\omega^{\prime}$ of $(N, \mathcal{W})$ such that $\omega^{\prime} \leq \omega$. If $\omega \leq \omega^{\prime}$ for every integral representation $\omega^{\prime}$ of $(N, \mathcal{W})$, then is the minimum integral representation of $(N, \mathcal{W})$. A representation is normalized if $\sum_{i \in N} \omega_{i}=1$.

In a constant-sum weighted majority game $(N, \mathcal{W})$, an imputation $x$ is a normalized representation of $(N, \mathcal{W})$ if and only if $q(x):=\underset{S \in \mathcal{W}_{m}}{\operatorname{Min}} x(S)>\frac{1}{2}$. Peleg (1968) has proved that any imputation in the least core of a constant-sum weighted majority game $(N, \mathcal{W})$ is a normalized representation of $(N, \mathcal{W})$; if $(N, \mathcal{W})$ is moreover homogeneous, then the nucleolus is the unique normalized homogeneous representation of $(N, \mathcal{W})$ which assigns zero to each null player. The nucleolus has rational coordinates, i.e. can be written as $x^{*}((N, \mathcal{W}))=\frac{\omega^{*}}{\omega^{*}(N)}$ where the $\omega_{i}^{*}$ for $i \in N$ are integers whose greatest common divisor

\footnotetext{
${ }^{31}$ See Von Neumann and Morgenstern (1944), Shapley (1962) and Taylor and Zwicker (1999).

${ }^{32}$ Some authors use the term strong for constant sum.
} 
is 1 . Peleg proves that if $(N, \mathcal{W})$ is a constant-sum weighted majority game then $\omega^{*}$ is a minimal integral representation if and only if $\omega^{*}(N)=2 q\left(\omega^{*}\right)-1$; if the game is moreover homogeneous $\omega^{*}$ is always the minimum integral representation. Sudhölter (1996) proved that if $(N, \mathcal{W})$ is a weighted majority game, then the modiclus is a normalized representation of $(N, \mathcal{W})$. Ostman (1987) and Rosenmüller (1987) showed that every homogeneous (not necessarily strong) weighted majority game has a unique minimal integral representation and this representation is homogeneous. Sudhölter (1996) proved that, up to normalization, this representation coincides with the modiclus.

These results point out the existence of relationships between the nucleolus and the set of minimal integral representations. Note however that the nucleolus need not be a representation at all if the game is not constant-sum. Even for constant-sum games, if the game is not homogeneous $\omega^{*}$ need not be a minimal integral representation. Peleg (1968) showed that $\omega^{*}$ is not a minimal integral representation for a game with $n=12$ and two minimal integral representations that appears in Isbell (1959). Isbell (1969) provides an example of a game with $n=19$ and a minimum integral representation $\underline{\omega}$ such that $\omega^{*} \neq \underline{\omega}$.

Krohn and Sudhölter (1995) proved that if $(N, \mathcal{W})$ is a constant-sum weighted majority game and $n \leq 8$, then $L C(N, V)=N_{u}(N, V)$ which coincides with the unique normalized minimal integral representation of $(N, \mathcal{W})$. For $n=9$, the result holds with the exception of 14 games which have exactly two minimal representations differing on one type of players. In 12 of these games both representations are the extreme points of the least core and the nucleolus is the midpoint of these representations. In the remaining two cases, no normalized representation is contained in the least core though the set is a singleton (i.e. coincides with the nucleolus). Freixas, Molinero and Roura (2007) show that these 14 games have a minimum symmetric integral representation, but this is not guaranteed for $n=10$.

If we do not require the game to be constant-sum, Freixas and Molinero (2009) found that all weighted majority games have a unique minimal integral representation for $n \leq 7$. For $n=8$ there are 154 weighted majority games without a minimum integral representation, though all of them have a minimum symmetric integral representation. Freixas and Molinero (2010) contains examples of games where $n=9$ without a minimum symmetric integral representation.

The enumeration of all simple games or subclasses like weighted majority games is important for the combinatorial optimization conducted in our paper. Constant-sum simple games were enumerated by von Neumann and Morgenstern (1944) for $n \leq 5$ and Gurk and Isbell (1959) for $n=6$. Isbell (1959) lists the 135 constant-sum weighted majority games for $n \leq 7$ together with their unique minimal integral representations; 38 of those games are homogeneous. Table 17 below is taken from Krohn and Sudhölter (1995), except for the number of directed games and of weighted majority games for $n=9$ which is taken from Kurz (2012). Note that Krohn and Sudhölter do not assume that $\varnothing \notin \mathcal{W}, N \in \mathcal{W}$ or that the game is proper.

The enumeration of all simple games (including the two constant ones attached to $V(\varnothing)=1$ and $V(N)=0$ ) is known as Dedekind's problem. Table 18 below reproduces the enumeration for games with $n \leq 6$.

The enumeration of all constant-sum simple games (also called maximal intersecting 
Table 17:

\begin{tabular}{llllllllll}
\hline \hline $\mathbf{n}$ & $\mathbf{1}$ & $\mathbf{2}$ & $\mathbf{3}$ & $\mathbf{4}$ & $\mathbf{5}$ & $\mathbf{6}$ & $\mathbf{7}$ & $\mathbf{8}$ & $\mathbf{9}$ \\
\hline \# directed games & 3 & 5 & 10 & 27 & 119 & 1173 & 44315 & 161175190 & 284432730176 \\
\# weighted majority games & 3 & 5 & 10 & 27 & 119 & 1113 & 29375 & 2730166 & 989913346 \\
\# constant-sum directed games & 1 & 1 & 2 & 3 & 7 & 21 & 135 & 2470 & 319124 \\
\# constant-sum weighted games & 1 & 1 & 2 & 3 & 7 & 21 & 135 & 2470 & 175428 \\
\# homogeneous games & 1 & 3 & 8 & 23 & 76 & 293 & 1307 & 6642 & 37882 \\
\hline
\end{tabular}

Table 18:

\begin{tabular}{lllllll}
\hline \hline $\mathbf{n}$ & $\mathbf{1}$ & $\mathbf{2}$ & $\mathbf{3}$ & $\mathbf{4}$ & $\mathbf{5}$ & $\mathbf{6}$ \\
\hline \# simple games & 3 & 6 & 20 & 168 & 7581 & 7828354 \\
\hline
\end{tabular}

families of sets) has also attracted attention. Table 19 below, extracted from Loeb and Conway (2000), reproduces the enumeration for games with $n \leq 8$.

Table 19:

\begin{tabular}{lllllllll}
\hline \hline $\mathbf{n}$ & $\mathbf{1}$ & $\mathbf{2}$ & $\mathbf{3}$ & $\mathbf{4}$ & $\mathbf{5}$ & $\mathbf{6}$ & $\mathbf{7}$ & $\mathbf{8}$ \\
\hline \# constant-sum simple games & 1 & 2 & 4 & 12 & 81 & 2646 & 1422564 & 229809982112 \\
\hline
\end{tabular}

The enumerations in tables 18 and 19 count games which are isomorphic. Otherwise the numbers decrease in a significant way as illustrated in table 20 below for games with $n \leq 7$.

Table 20:

\begin{tabular}{llllllll}
\hline \hline $\mathbf{n}$ & $\mathbf{1}$ & $\mathbf{2}$ & $\mathbf{3}$ & $\mathbf{4}$ & $\mathbf{5}$ & $\mathbf{6}$ & $\mathbf{7}$ \\
\hline \# isomorphism classes of constant-sum simple games & 1 & 1 & 2 & 3 & 7 & 30 & 716 \\
\hline
\end{tabular}

We may also limit the enumeration to games where some players are always treated similarly (the set of players is partitioned into a number of types where two players of the same type are perfect substitutes in the simple game). Freixas, Molinero and Roura (2009) and Kurz and Tautenhahn (2010) have derived formulas to enumerate all such simple games.

\section{References}

[1] Algaba, E. Bilbao, J.M and J.R. Fernández (2007) "The Distribution of Power in the European Constitution", European Journal of Operational Research, 176, 1752-1766.

[2] Alon, N. Edelman, P.H. (2010) "The Inverse Banzhaf Problem", Social Choice and Welfare, 34, 371-377.

[3] Banks, J.S. (2000) "Buying Supermajorities in Finite Legislatures", American Political Science Review, 94, 677-681. 
[4] Banks, J.S. and J. Duggan (2000) "A Bargaining Model of Collective Choice", American Political Science Review, 94, 73-88.

[5] Banzhaf, J.F. III. (1965) "Weighted Voting Doesn't Work : A Mathematical Analysis", Rutgers Law Review, 19, 317-343.

[6] Barberà, S. and M.O. Jackson (2006) "On the Weights of Nations : Assigning Voting Weights in a Heterogeneous Union", Journal of Political Economy, 114, 317-339.

[7] Baron, D. P. and J. A. Ferejohn (1989) "Bargaining in Legislatures", American Political Science Review, 83, 1181-1206.

[8] Barr, J. and F. Passarelli (2009) "Who Has the Power in the EU?", Mathematical Social Sciences, 57, 339-366.

[9] Beisbart, C., Bovens, L. and S. Hartmann (2005) "A Utilitarian Assessment of Alternative Decision Rules in the Council of Ministers", European Union Politics, 6, 395-418.

[10] Beisbart, C. and L. Bovens (2007) "Welfarist Evaluations of Decision Rules for Boards of Representatives", Social Choice and Welfare, 29, 581-608.

[11] Beisbart, C. and S. Hartmann (2010) "Welfarist Evaluations of Decision Rules under Interstate Utility Dependencies", Social Choice and Welfare, 34, 315-344.

[12] Bilbao, J.M., Fernández, J.R., Jiménez, J.J. and J.J. López (2002) "Voting Power in the European Union Enlargement", European Journal of Operational Research, 143, 181-196.

[13] Coleman, J.S. (1971) "Control of Collectivities and the Power of a Collectivity to Act", in Social Choice (ed. by B. Lieberman). New York: Gordon and Breach.

[14] Derks, J. and J. Kuipers (1997)."Implementing the Simplex Method for Computing the Prenucleolus of Transferable Utility Games". Unpublished manuscript.

[15] Diermeier, D. and R.B. Myerson. (1999) "Bicameralism and its Consequences for the Internal Organization of Legislatures". American Economic Review, 89, 1182-1196.

[16] Dubey, P. (1975) "On the Uniqueness of the Shapley Value". International Journal of Game Theory, 4, 131-139.

[17] Eraslan, H. (2002) "Uniqueness of Stationary Equilibrium Payoffs in the Baron-Ferejohn Model", Journal of Economic Theory, 103, 11-30.

[18] Eraslan, H., A. McLennan (2006) "Uniqueness of Stationary Equilibrium Payoffs in Coalitional Bargaining", Mimeo.

[19] Felsenthal, D. S. and M. Machover (1998) The Measurement of Voting Power. Theory and Practice, Problems and Paradoxes, Cheltenham: Edward Elgar. 
[20] Felsenthal, D. S. and M. Machover (2001) "The Treaty of Nice and Qualified Majority Voting", Social Choice and Welfare 18, 431-464.

[21] Felsenthal, D. S. and M. Machover (2004). "Analysis of QM Rules in the Draft Constitution for Europe proposed by the European Convention, 2003", Social Choice and Welfare 23, 1-25.

[22] Freixas, J., Molinero, X. and S. Roura (2007) "Minimal Representations for Majority Games", in Computations and Logic in the Real World, Lecture Notes in Computer Science, Vol 4497, Springer Verlag, 297-306.

[23] Freixas, J. and X. Molinero (2009) "On the Existence of Minimum Integer Representation for Weighted Voting Systems", Annals of Operations Research, 166, 243-260.

[24] Freixas, J., Molinero, X. and S. Roura (2009) "A Fibonnaci Sequence for Linear Structures with Two Types of Components", Mimeo.

[25] Freixas, J. and X. Molinero (2010) "Weighted Games without a Unique Minimal Representation in Integers", Optimization Methods and Software, 25, 203-215.

[26] Groseclose, T. and J.M. Snyder. (1996) "Buying Supermajorities", American Political Science Review, 90, 303-315.

[27] Gurk, H.M. and J.R. Isbell (1959) "Simple Solutions" in Annals of Mathematics Studies, Contributions to the Theory of Games IV, Volume 40, Princeton University Press, 247265.

[28] Isbell, J.R. (1959) "On the Enumeration of Majority Games", Mathematical Tables and Other Aids to Computation, 13, 21-28.

[29] Isbell, J.R. (1969) "A Counterexample in Weighted Majority Games", Proceedings of the American mathematical Society, 20, 590-592.

[30] Kalandrakis, T. (2006) "Proposal Rights and Political Power", American Journal of Political Science, 50, 441-448.

[31] Kaniovski, S. and D. Leech (2009) "A Behavioral Power Index", Public Choice, 141, 17-29.

[32] Krohn, I. and P. Sudhölter (1995) "Directed and Weighted Majority Games", Mathematical Methods of Operations Research, 42, 189-216.

[33] Kurz, S. (2012) "On Minimum Sum Representations for Weighted Voting Games", forthcoming in Annals of Operations Research.

[34] Kurz, S. and Tautenhahn (2010) "On Dedekind's Problem for Complete Simple Games", Mimeo. 
[35] Kurz, S., Maaser, N. and Napel, S. (2011). "On the Egalitarian Weights of Nations", Unpublished manuscript.

[36] Laruelle, A. and F. Valenciano (2008) Voting and Collective Decision Making, Cambridge University Press.

[37] Laruelle, A. and M. Widgrén (1998) " Is the Allocation of Voting Power among the EU States Fair?", Public Choice, 94, 317-339.

[38] Le Breton, M. and V. Zaporozhets (2010) "Sequential Legislative Lobbying under Political Certainty", Economic Journal, 120(543), 281-312.

[39] Le Breton, M., Sudhölter, P. and V. Zaporozhets (2010) "Sequential Legislative Lobbying", DP No. 8/2009, Department of Business and Economics, University of Southern Denmark.

[40] Leech, D. (2002) "Designing the Voting System for the Council of Ministers of the European Union", Public Choice, 113, 437-464.

[41] Loeb, D.E. and A.R. Conway (2000) "Voting Fairly: Transitive Maximal Intersecting Family of Sets", Journal of Combinatorial Theory, Series A, 91, 386-410.

[42] Maschler, M., Peleg, B., Shapley, L.S. (1979) "Geometric Properties of the Kernel, Nucleolus, and Related Solution Concepts", Mathematics of Operations Research, 303338.

[43] Matsui, T. and Y. Matsui (2000) "A Survey of Algorithms for Calculating Power Indices of Weighted Majority Games", Journal of the Operations Research Society of Japan, $43,71-85$.

[44] Maaser, N. and S. Napel (2007) "Equal Representation in Two-Tier Voting Systems", Social Choice and Welfare, 28, 401-420.

[45] Monroe, B.L. (1994) "Disproportionality and Malapportionment: Measuring Electoral Inequity", Electoral Studies, 13, 132-149.

[46] Montero, M. (2005) "On the Nucleolus as a Power Index", Homo Oeconomicus, 4, 551-567.

[47] Montero, M. (2006) "Noncooperative Foundations of the Nucleolus in Majority Games", Games and Economic Behavior, 54, 380-397.

[48] Montero, M. (2007) "The Paradox of New Members in the Council of Ministers: A Noncooperative Approach", CeDEx Discussion Paper, No 2007-12.

[49] Napel, S. and M. Widgrén (2004) "Power Measurement as Sensitivity Analysis", Journal of Theoretical Politics, 16, 517-538. 
[50] Napel, S. and M. Widgrén (2006) "The Inter-Institutional Distribution of Power in EU Codecision", Social Choice and Welfare, 27, 129-154.

[51] Napel, S. and M. Widgrén (2011) "Strategic versus Non-Strategic Voting Power in the EU Council of Ministers: The Consultation Procedure", Social Choice and Welfare, 37, 511-541.

[52] Ostmann, A. (1987) "On the Minimal Representation of Homogeneous Games", International Journal of Game Theory, 16, 69-81.

[53] Owen, G. (1995) Game Theory, California: Academic Press.

[54] Pajala, A. (2005) "Maximal Proportionality between Votes and Voting Power: The Case of the Council of the European Union", Tyescript.

[55] Peleg, B. (1968) "On Weights of Constant-Sum Majority Games", SIAM Journal on Applied Mathematics, 16, 527-532.

[56] Peleg, B. and P. Sudhölter (2003) Introduction to the Theory of Cooperative Games, Boston, Kluwer Academic Publishers.

[57] Penrose, L. S. (1946) "The Elementary Statistics of Majority Voting", Journal of the Royal Statistical Society, 109, 53-57.

[58] Roth, A. (1977) "The Shapley Value as a von Neumann-Morgenstern Utility", Econometrica, 45, 657-664.

[59] Rosenmüller, J. (1987) "Homogeneous Games : Recursive Structure and Computation", Mathematics of Operations Research, 12, 309-330.

[60] Schmeidler, D. (1969) "The Nucleolus of a Characteristic Function Game", SIAM Journal on Applied Mathematics, 17, 1163-1170.

[61] Schmidtchen, D. and B. Steunenberg (2011) "On the Possibility of a Preference-based Power Index: The Strategic Power Index Revisited", Typescript.

[62] Shapley, L.S. (1953) "A Value for n-Person Games", Annals of Mathematical Studies $28,307-317$.

[63] Shapley, L.S. (1962) "Simple Games: An Outline of the Descriptive Theory", Behavioral Sciences, 7, 59-66.

[64] Shapley, L.S. and M. Shubik (1954) "A Method for Evaluating the Distribution of Power in a Committee System", American Political Science Review, 48, 787-792.

[65] Snyder, J.M., Ting, M. M. and S. Ansolabehere (2005) "Legislative Bargaining under Weighted Voting", American Economic Review, 95, 981-1004. 
[66] Steunenberg, B., Schmidtchen, D. and C. Koboldt (1999) "Strategic Power in the European Union : Evaluating the Distribution of Power in Policy Games", Journal of Theoretical Politics, 11, 339-366.

[67] Sudhölter, P. (1996) "The Modified Nucleolus as Canonical Representation of Weighted Majority Games", Mathematics of Operations Research, 21, 734-756.

[68] Sudhölter, P. (1997) "The Modified Nucleolus: Properties and Axiomatizations", International Journal of Game Theory 26, 147-182.

[69] Taylor, A.D. and W.S. Zwicker (1999) Simple Games, Princeton University Press.

[70] Tsebelis, G. (1994) "The Power of the European Parliament as a Conditional Agenda Setter", American Political Science Review, 88, 128-142.

[71] Van Puyenbroeck, T. (2008) "Proportional Representation, Gini Coefficients, and the Principle of Transfers", Journal of Theoretical Politics, 20, 498-526.

[72] Von Neumann, J. and O. Morgenstern. (1944) Theory of Games and Economic Behavior, Princeton: Princeton University Press.

[73] Wolsey, L.A. (1976) "The Nucleolus and Kernel of Simple Games or Special Valid Inequalities for 0-1 Linear Integer programs", International Journal of Game Theory, 5, 225-238.

[74] Yan, H. (2009) "Uniqueness in Random-Proposer Multilateral Bargaining", International Game Theory Review, 11, 407-417.

[75] Young, H.P. (1978a) "A Tactical Lobbying Game" in Game Theory and Political Science, Ordeshook, P.C. (Ed), New York University Press, New York.

[76] Young, H.P. (1978b) "The Allocation of Funds in Lobbying and Campaigning", Behavioral Science, 23, 21-31.

[77] Young, H.P. (1978c) "Power, Prices, and Incomes in Voting Systems", Mathematical Programming, 14, 129-148.

[78] Życzkowski, K., and W. Słomczyński (2004) "Voting in the European Union: The Square Root System of Penrose and a Critical Point". Preprint at http://arxiv.org/abs/condmat/0405396. 